\title{
Relative Similarity Programming Model for Uncertain Multiple Attribute Decision-Making Objects and Its Application
}

\author{
Zhili Huang $\mathbb{D}^{1},{ }^{1}$ Qinglan Chen $\mathbb{D}^{1},{ }^{1}$ Liu Chen $\mathbb{D},{ }^{1}$ and Qinyuan Liu $\mathbb{D}^{2}$ \\ ${ }^{1}$ School of Economics and Management, Xiamen University of Technology, Xiamen 361024, China \\ ${ }^{2}$ Department of Computer Science and Technology, Tongji University, Shanghai 200092, China \\ Correspondence should be addressed to Qinglan Chen; blue777@163.com and Qinyuan Liu; liuqy@tongji.edu.cn
}

Received 6 November 2020; Revised 4 December 2020; Accepted 13 February 2021; Published 9 March 2021

Academic Editor: Yi-Zhang Jiang

Copyright (c) 2021 Zhili Huang et al. This is an open access article distributed under the Creative Commons Attribution License, which permits unrestricted use, distribution, and reproduction in any medium, provided the original work is properly cited.

This paper is concerned with the uncertain multiattribute decision-making (UMADM) of which the attribute value is triangular fuzzy number. Firstly, the max-relative similarity degree and min-relative similarity degree of alternative decision-making objects are given based on the relative similarity degree of triangular fuzzy number, the advantage relation theories to comparative relative similarity degree of triangular fuzzy number are proposed, and some good properties, relations, and conclusions are derived. Secondly, in order to determine the attribute weight vector, a triangular fuzzy number-based decision-making object relative similarity programming model is established with the help of maximizing possibility degree algorithm rules in the cooperative game theory. Subsequently, by aggregating the comparison overall relative similarity degree values of all decision-making objects, we could pick over and sort the set of alternative objects and gather a new model algorithm for the relative similarity programming of triangular fuzzy number-based multiple attribute decision-making alternatives. Finally, an example is given to illustrate the feasibility and practicability of the model algorithm presented in this paper.

\section{Introduction}

Uncertain multiattribute decision-making (UMADM) is one of the main contents of decision analysis, management, control engineering, and system engineering [1], and the acquisition of attribute weight information is a hot research field in UMADM. The research on uncertain multiattribute decision-making mainly focuses on supplier selection, benefit evaluation of logistics network, transportation optimization, enterprise production benefit and risk evaluation, multiagent system, waste management site selection, logistics support evaluation, and other disciplines [2-7]. Due to objective factors, complexity, randomness, uncertainty and disturbance of surrounding environment, subjectivity, fuzziness and preference of thinking, and empirical, stereotyped, and limited of cognition, it is usually impossible or difficult for people to express and describe the specific attribute utility measure information with accurate numbers when they are dealing with solving UMADM problems or objects, especially those with outstanding development and innovation.
Therefore, their quantification usually takes the form of interval numerical value, multivariate interval value, triangular fuzzy number value, or uncertain language value. The research on this kind of UMADM problem has attracted many experts and scholars at home and abroad. Van Laarhoven, a Dutch expert who is one of the famous scholars, put forward the comparative judgment method of describing fuzzy uncertain information with triangular fuzzy number [8]. Compared with the comparative judgment $[9,10]$, the comparative judgment of triangular fuzzy number can more consistently meet the objective uncertainty of the environment and the subjective fuzziness of logical thinking and more scientifically and accurately describe the quality judgment of uncertain multiple attribute decision-making objects. The acquisition of attribute optimization weight information is an important research content in UMADM problem. It is easy to find that most scholars focus on the following weight measurement methods: VIKOR method [11], AHP-VIKOR method [12], relative dominance relation method [13], relative similarity degree relation method $[14,15]$, quadratic 
programming and relative superiority degree method [16], grey target decision-making method [17], grey relational analysis method [18], Grey relation-projection pursuit dynamic cluster method [19], similarity measurement of cloud model [20], rough hesitant fuzzy method based on rough sets [21], generalized complex intuitionistic fuzzy aggregation operators [22], robust averaging-geometric aggregation operators for complex intuitionistic fuzzy sets [23], Pythagorean fuzzy aggregation operators [24, 25], Pythagorean hybrid weighted aggregation operators with Pythagorean fuzzy numbers [26], distance measures of complex Pythagorean fuzzy sets [27], q-rung orthopair fuzzy point weighted aggregation operators [28], power aggregation operators for complex q-rung orthopair fuzzy sets [29], aggregation operators based on complex q-rung orthopair uncertain linguistic information [30], aggregation operators based on complex interval-valued q-rung orthopair fuzzy sets [31], and so on. Then, the relevant decision information is aggregated by combining the respective proposed models and algorithms to pick over and sort the set of alternative objects. Although literature [15] proposed a method to determine the attribute weight based on relative similarity degree relation with the help of maximizing possibility degree algorithm in cooperative game, it failed to give a specific solution process to determine the attribute weight. In the literature [32], maximizing possibility degree algorithm rules in the cooperative game theory is adopted to obtain the attribute optimization weight vector: from the perspective of picking over and sorting decision-making objects alternatives, the greater the total attribute comparative possibility degree value of decision-making objects is, the greater the assignment of attribute weight is, and the smaller the total attribute comparative possibility degree value of decision-making objects is, the smaller the assignment of attribute weight is; therefore, the comprehensive attribute value difference of all alternative decision objects assembled and merged under the optimal weighting is further expanded. On the other hand, [33] gives a too simple and loose deviation formula to express the definition of similarity based on the similarity principle, which aggregates and merges different attribute data types. For example, in the special case that the length of triangular fuzzy numbers degenerated into real numbers or interval numbers is 0 , it is easy to cause the accumulation of errors in the decision-making process, which eventually leads to the failure of information fusion and the error of picking over and sorting decision-making objects alternatives.

For UMADM problem whose attribute value is triangular fuzzy number, the paper gives two new definitions: max- and min-relative similarity degree of triangular fuzzy number and decision-making object of max-relative similarity degree and min-relative similarity degree from the basic idea of measuring best-worst scaling between alternative decision objects and calculating the similarity degree of triangular fuzzy number, which better integrates the geometric position relationship of decision object attribute utility measure data sequence, the close level of curve shape and the similarity degree, and is convenient to characterize the connection and difference between decision objects, thus overcoming the problems in literature [33]. On the basis of the above definition, we introduce a new attribute weighting rule based on the relative similarity programming model of decision-making objects: after unifying the incommensurability of attribute data, the comprehensive relative similarity degree of evaluation values of decision objects under the same attribute utility measure is higher (the difference of attribute measure values is smaller), and this attribute has less influence on picking over and sorting alternatives, so the degree of emphasis should be reduced in the decisionmaking process, and the measure value of attribute weight should be reduced. Especially, under the same attribute utility measure, the comprehensive relative similarity of evaluation values reaches the highest; namely, it is equal to 1 (there is no difference in attribute measure values); then this attribute will have no effect on picking over and sorting decision-making alternatives, and the measure value of attribute weight should be zero. On the contrary, the comprehensive relative similarity degree of evaluation values of decision-making alternatives under the same attribute utility measure is relatively low (the difference of attribute measurement values is large), so the attribute has a greater impact on picking over and sorting the decision-making alternatives, the degree of emphasis should be increased, and the measurement value of attribute weight should be increased in the decision-making process, because the changes of attribute utility measure data is the fundamental reason that causes the change of the order and selection of decision objects. In this paper, using the abovementioned weighting thought and the advantage relation theories to comparative relative similarity degree of triangular fuzzy number, under the attribute weight assignment based on decision making objects relative similarity programming model, the comprehensive relative similarity degree value between decisionmaking objects is reduced, and the sort difference between decision-making objects is increased, by reconciling the information of all decision-making objects. According to the overall relative similarity degree value between each decision object and the ideal optimal object in the set of decision objects to pick over and sort, then decision-making object relative similarity programming model algorithm of UMADM is obtained.

In a word, the main work of this paper is to fully consider the influence of the relative similarity measurement information between attribute values on the attribute itself in the evaluation process such as UMADM and redesign and construct a new decision-making object relative similarity programming model (DMORSPM) based on the triangular fuzzy number attribute value comparative similarity relationship to obtain more optimized attribute weighting formula. In order to solve the problem of low overall discrimination after the feature, information of decisionmaking object is gathered and fused. The main advantage of TFN-DMORSPM algorithm proposed in this paper is that it can expand the discrimination between decision-making objects under the condition of processing data information 
and very high similarity degree of attribute measurement values, facilitate the accurate calculation and aggregation of data, and promote the optimal decision-making and sorting of decision-making objects.

\section{The Advantage Relation Theories to Comparative Relative Similarity Degree of Triangular Fuzzy Number}

\subsection{Comparative Relative Similarity Degree of Triangular Fuzzy Number}

Definition 1. If $\tilde{x}=\left[x^{L}, x^{M}, x^{U}\right]=\left\{x \mid 0<x^{L} \leq x^{M} \leq x^{U}, x\right.$, $\left.x^{L}, x^{M}, x^{U} \in R\right\}, \tilde{x}$ is a triangular fuzzy number (TFN) $[7,8,15]$, where $x^{L}$ and $x^{U}$ are the lower and upper bounds of triangular fuzzy number $\tilde{x}$, which are generally called small elements and large elements. $x^{M}$ is the median value supported by triangular fuzzy number $\tilde{x}$ (i.e., information tendency preference value, which usually refers to the number with the highest probability of being selected in this interval), generally called special element. If $\tilde{x}$ also satisfies
$0<x^{L} \leq x^{M} \leq x^{U}<1$, then $\tilde{x}$ is called a normalized triangular fuzzy number. If $x^{L}=x^{M}$ or $x^{M}=x^{U}, \tilde{x}=\left[x^{L}, x^{M}, x^{U}\right]$ degenerates into an interval number $\tilde{x}=\left[x^{M}, x^{U}\right]$ or $\tilde{x}=\left[x^{L}, x^{M}\right]$, in particular, if $x^{L}=x^{M}=x^{U}$, then $\tilde{x}$ is reduced to an ordinary positive real number; that is, $\tilde{x}=x^{L}=x^{M}=x^{U}$.

For the convenience of follow-up analysis, the operation rules of triangular fuzzy numbers are introduced: let $\tilde{x}=\left[x^{L}, x^{M}, x^{U}\right], \tilde{y}=\left[y^{L}, y^{M}, y^{U}\right]$, and then we have

(i) Rule 1: $\tilde{x}+\tilde{y}=\left[x^{L}+y^{L}, x^{M}+y^{M}, x^{U}+y^{U}\right]$

(ii) Rule 2: $(1 / \tilde{x})=\left[\left(1 / x^{U}\right),\left(1 / x^{M}\right),\left(1 / x^{L}\right)\right], x^{L}, x^{M}, x^{U}$ $\neq 0$

(iii) Rule 3: $k \tilde{x}=\left[k x^{L}, k x^{M}, k x^{U}\right], k \geq 0$

(iv) Rule 4: $\tilde{x} \times \tilde{y}=\left[x^{L} y^{L}, x^{M} y^{M}, x^{U} y^{U}\right]$

(v) Rule 5: if and only if $x^{L}=y^{L}, x^{M}=y^{M}, x^{U}=y^{U}$, then $\tilde{x}=\tilde{y}$

Definition 2. Let two arbitrary triangular fuzzy numbers $\widetilde{x}=$ $\left[x^{L}, x^{M}, x^{U}\right]$ and $\tilde{y}=\left[y^{L}, y^{M}, y^{U}\right]$. Then,

$$
r s_{\mathrm{Max}}(\tilde{x}, \tilde{y})=\frac{\left(x^{L} y^{L}+x^{M} y^{M}+x^{U} y^{U}\right)^{(1 / 2)}}{\max \left\{\left[\left(x^{L}\right)^{2}+\left(x^{M}\right)^{2}+\left(x^{U}\right)^{2}\right]^{(1 / 2)},\left[\left(y^{L}\right)^{2}+\left(y^{M}\right)^{2}+\left(y^{U}\right)\right]^{2(1 / 2)}\right\}}
$$

is called Max-relative similarity degree between triangular

fuzzy numbers $\tilde{x}$ and $\tilde{y}$, or

$$
r s_{\operatorname{Min}}(\tilde{x}, \tilde{y})=\frac{\min \left\{\left[\left(x^{L}\right)^{2}+\left(x^{M}\right)^{2}+\left(x^{U}\right)^{2}\right]^{(1 / 2)},\left[\left(y^{L}\right)^{2}+\left(y^{M}\right)^{2}+\left(y^{U}\right)^{2}\right]^{(1 / 2)}\right\}}{\left(x^{L} y^{L}+x^{M} y^{M}+x^{U} y^{U}\right)^{(1 / 2)}},
$$

is called Min-relativesimilarity degree between triangular fuzzy numbers $\tilde{x}$ and $\tilde{y}$. Apparently, the greater the $r s_{\text {Max }}(\tilde{x}, \tilde{y})$ or $r s_{\text {Min }}(\tilde{x}, \tilde{y})$, the greater the similarity degree between $\tilde{x}$ and $\tilde{y}$. In special cases, when $r s_{\mathrm{Max}}(\tilde{x}, \tilde{y})=1$ or $r s_{\text {Min }}(\tilde{x}, \tilde{y})=1$, one has $\tilde{x}=\tilde{y}$; that is, the triangular fuzzy number $\tilde{x}$ is completely similar to $\tilde{y}$.

According to the max-relative and min-relative similarity degree of triangular fuzzy numbers given by Definition 2 , the following properties can be revealed.

Theorem 1. Let any given triangular fuzzy number be set as $\tilde{x}=\left[x^{L}, x^{M}, x^{U}\right], \tilde{y}=\left[y^{L}, y^{M}, y^{U}\right], \widetilde{z}=\left[z^{L}, z^{M}, z^{U}\right]$; then we have the following:

(1) Boundedness, $0 \leq r s_{\text {Max }}(\tilde{x}, \tilde{y}) \leq 1,0 \leq r s_{\text {Min }}(\tilde{x}, \tilde{y}) \leq 1$

(2) Reflexivity, $r s_{\text {Max }}(\tilde{x}, \tilde{x})=1, r s_{\text {Min }}(\tilde{x}, \tilde{x})=1$

(3) Symmetry property, $\quad r s_{\text {Max }}(\tilde{x}, \tilde{y})=r s_{\text {Max }}(\tilde{y}, \tilde{x})$, $r s_{\text {Min }}(\tilde{x}, \tilde{y})=, r s_{\text {Min }}(\widetilde{y}, \tilde{x})$
(4) Transitivity, $\quad r s_{\operatorname{Max}}(\tilde{x}, \tilde{y})=1, \quad \vee, \quad r s_{\text {Min }}(\tilde{x}, \tilde{y})=1$, $r s_{\text {Max }}(\tilde{y}, \widetilde{z})=1, \vee, r s_{\text {Min }}(\tilde{y}, \tilde{z})=1$, that is, if $\tilde{x}$ and $\tilde{y}$ are completely similar, $\widetilde{y}$ and $\tilde{z}$ are completely similar, then $\tilde{x}$ and $\tilde{z}$ are completely similar

(5) If $r s_{\text {Max }}(\tilde{x}, \tilde{y}) \leq r s_{\text {Max }}(\tilde{x}, \tilde{z}), \vee, \quad r s_{\text {Min }}(\tilde{x}, \tilde{y}) \leq r s_{\text {Min }}$ $(\tilde{x}, \tilde{z})$, then $\widetilde{z}$ is closer to $\tilde{x}$ compared with $\tilde{y}$; if $r s_{\text {Max }}(\tilde{x}, \tilde{y}) \leq, r s_{\text {Max }}(\tilde{z}, \tilde{y}), \vee, r s_{\text {Min }}(\tilde{x}, \tilde{y}) \leq r s_{\text {Min }}(\tilde{z}$, $\tilde{y})$, then $\widetilde{z}$ is closer to $\tilde{y}$ compared with $\tilde{x}$

According to the definition of max-relative and minrelative similarity degree of triangular fuzzy numbers, it is easy to prove that the conclusion of Theorem 1 is valid. The process of proof is omitted.

Definition 3. Let alternative decision-making objects be composed of triangular fuzzy number sequence set as $X=\left\{\tilde{x}_{1}, \tilde{x}_{2}, \ldots, \tilde{x}_{m}\right\}, Y=\left\{\tilde{y}_{1}, \tilde{y}_{2}, \ldots, \tilde{y}_{m}\right\}$. Then, one has 


$$
\operatorname{RS}_{\text {Max }}(X, Y)=\frac{\sum_{i=1}^{m}\left(x_{i}^{L} y_{i}^{L}+x_{i}^{M} y_{i}^{M}+x_{i}^{U} y_{i}^{U}\right)^{(1 / 2)}}{\sum_{i=1}^{m} \max \left\{\left[\left(x_{i}^{L}\right)^{2}+\left(x_{i}^{M}\right)^{2}+\left(x_{i}^{U}\right)^{2}\right]^{(1 / 2)},\left[\left(y_{i}^{L}\right)^{2}+\left(y_{i}^{M}\right)^{2}+\left(y_{i}^{U}\right)^{2}\right]^{(1 / 2)}\right\}},
$$

where $\operatorname{RS}_{\text {Max }}(X, Y)$ is called max-relative similarity degree based decision-making objects $X$ and $Y$,and

$$
\operatorname{RS}_{\text {Min }}(X, Y)=\frac{\sum_{i=1}^{m} \min \left\{\left[\left(x_{i}^{L}\right)^{2}+\left(x_{i}^{M}\right)^{2}+\left(x_{i}^{U}\right)^{2}\right]^{(1 / 2)},\left[\left(y_{i}^{L}\right)^{2}+\left(y_{i}^{M}\right)^{2}+\left(y_{i}^{U}\right)^{2}\right]^{(1 / 2)}\right\}}{\sum_{i=1}^{m}\left(x_{i}^{L} y_{i}^{L}+x_{i}^{M} y_{i}^{M}+x_{i}^{U} y_{i}^{U}\right)^{(1 / 2)}},
$$

where $\operatorname{RS}_{\text {Min }}(X, Y)$ is called min-relative similarity degree based decision-making objects $X$ and $Y$.

By letting normalized triangular fuzzy number-based decision-making objects matrix $\widetilde{Z}=\left(\widetilde{z}_{i j}\right)_{n \times m}$, where $\widetilde{z}_{i j}=\left[z_{i j}^{L}, z_{i j}^{M}, z_{i j}^{U}\right], i \in N, j \in M$, we can give the following definition.
Definition 4. $Z^{*+}=\left\{\widetilde{z}_{1}^{*+}, \widetilde{z}_{2}^{*+}, \ldots, \widetilde{z}_{m}^{*+}\right\}$ is the triangular fuzzy number-based positive ideal decision-making objects composed of positive ideal point sequence, where

$$
\tilde{z}_{j}^{*+}=\left[z_{j}^{*+L}, z_{j}^{*+M}, z_{j}^{*+U}\right]=\left[\max _{i}\left(z_{i j}^{L}\right), \max _{i}\left(z_{i j}^{M}\right), \max _{i}\left(z_{i j}^{U}\right)\right], \quad(j=1,2, \ldots, m),
$$

and $Z^{*-}=\left\{\widetilde{z}_{1}^{*-}, \widetilde{z}_{2}^{*-}, \ldots, \widetilde{z}_{m}^{*-}\right\}$ is the triangular fuzzy number-based negative ideal decision-making objects composed of negative ideal point sequence

$$
\tilde{z}_{j}^{*-}=\left[z_{j}^{*-L}, z_{j}^{*-M}, z_{j}^{*-U}\right]=\left[\min _{i}\left(z_{i j}^{L}\right), \min _{i}\left(z_{i j}^{M}\right), \min _{i}\left(z_{i j}^{U}\right)\right], \quad(j=1,2, \ldots, m),
$$

2.2. The Advantage Relation to Comparative Relative Similarity Degree of Triangular Fuzzy Number. According to the concept of max-relative similarity degree and min-relative similarity degree of triangular fuzzy number, the definition and main results of the advantage relation of comparative relative similarity of triangular fuzzy number sequence and triangular fuzzy number are as follows.

Definition 5. Let any two triangular fuzzy numbers $\tilde{x}=\left[x^{L}, x^{M}, x^{U}\right]$ and $\tilde{y}=\left[y^{L}, y^{M}, y^{U}\right]$ and two triangular fuzzy number-based positive and negative ideal points $\tilde{z}^{*+}=\left[z^{*+L}, z^{*+M}, z^{*+U}\right]$ and $\tilde{z}^{*-}=\left[z^{*-L}, z^{*-M}\right.$, $\left.z^{*-U}\right]$; if

$r s_{\text {Max }}\left(\tilde{y}, \widetilde{z}^{*+}\right)>r s_{\text {Max }}\left(\widetilde{x}, \widetilde{z}^{*+}\right)$ or $r s_{\text {Min }}\left(\tilde{y}, \widetilde{z}^{*-}\right)<r s_{\text {Min }}\left(\widetilde{x}, \widetilde{z}^{*-}\right)$,

then the triangular fuzzy number $\tilde{y}$ is called $\tilde{y}$ superior to $\tilde{x}$, which is denoted as $\tilde{y}>\tilde{x}$. Obviously, the greater the max-relative similarity degree with the positive ideal point triangular fuzzy number or the smaller the minrelative similarity degree with the negative ideal point 
triangular fuzzy number is, the greater the dominance degree of the corresponding triangular fuzzy number is.

Theorem 2. If and only if the positive and negative ideal points of triangular fuzzy number are optimal decision points, then

$$
\begin{aligned}
\tilde{y}> & \tilde{x} \Longleftrightarrow r s_{\operatorname{Max}}\left(\tilde{y}, \tilde{z}^{*+}\right)>r s_{\operatorname{Max}}\left(\tilde{x}, \tilde{z}^{*+}\right) \vee r s_{\operatorname{Min}}\left(\tilde{y}, \tilde{z}^{*-}\right) \\
& <r s_{\operatorname{Min}}\left(\tilde{x}, \tilde{z}^{*-}\right) \\
q_{1}^{*+} & y^{L}+q_{2}^{*+} y^{M}+y^{U}>q_{1}^{*+} x^{L}+q_{2}^{*+} x^{M} \\
& +x^{U} \vee q_{1}^{*-} y^{L}+q_{2}^{*-} y^{M} \\
& +y^{U}>q_{1}^{*-} x^{L}+q_{2}^{*-} x^{M}+x^{U} \Longleftrightarrow \operatorname{among} q_{1}^{*+}=\frac{z^{*+L}}{z^{*+U}}, \\
q_{2}^{*+}= & \frac{z^{*+M}}{z^{*+U}}, \\
q_{1}^{*-}= & \frac{z^{*-L}}{z^{*-U}}, \\
q_{2}^{*-}= & \frac{z^{*-M}}{z^{*-U}} .
\end{aligned}
$$

Proof. Obviously, according to Definition 5,

$$
\begin{aligned}
\tilde{y} & >\widetilde{x} \\
& <r s_{\operatorname{Min}}\left(\tilde{x}, \tilde{z}^{*-}\right) .
\end{aligned}
$$

When positive and negative ideal points are optimal decision points, $\widetilde{z}^{*+}=\left[z^{*+L}, z^{*+M}, z^{*+U}\right]$ and $\tilde{z}^{*-}=\left[z^{*-L}, z^{*-M}, z^{*-U}\right]$ are the ideal point triangular fuzzy number; and hence we have

$$
\begin{aligned}
0 & <z^{*+L} \leq z^{*+M} \leq z^{*+U}, 0<z^{*-L} \leq z^{*-M} \leq z^{*-U}, \\
z^{*+L} & \geq \max \left\{x^{L}, y^{L}\right\} \\
z^{*+M} & \geq \max \left\{x^{M}, y^{M}\right\} \\
z^{*+U} & \geq \max \left\{x^{U}, y^{U}\right\} \\
z^{*-L} & \leq \min \left\{x^{L}, y^{L}\right\} \\
z^{*-M} & \leq \min \left\{x^{M}, y^{M}\right\} \\
z^{*-U} & \leq \min \left\{x^{U}, y^{U}\right\}
\end{aligned}
$$

and then we can get

$$
\begin{aligned}
q_{1}^{*+}= & \frac{z^{*+L}}{z^{*+U}} \leq 1, \\
q_{2}^{*+}= & \frac{z^{*+M}}{z^{*+U}} \leq 1, \\
q_{1}^{*-}= & \frac{z^{*-L}}{z^{*-U}} \leq 1, \\
q_{2}^{*-}= & \frac{z^{*-M}}{z^{*-U}} \leq 1, \\
& \cdot\left(z^{*+L}\right)^{2}+\left(z^{*+M}\right)^{2}+\left(z^{*+U}\right)^{2} \\
& \geq \max \left\{\left(x^{L}\right)^{2}+\left(x^{M}\right)^{2}+\left(x^{U}\right)^{2},\left(y^{L}\right)^{2}+\left(y^{M}\right)^{2}+\left(y^{U}\right)^{2}\right\}, \\
& \cdot\left(z^{*-L}\right)^{2}+\left(z^{*-M}\right)^{2}+\left(z^{*-U}\right)^{2} \\
& \leq \min \left\{\left(x^{L}\right)^{2}+\left(x^{M}\right)^{2}+\left(x^{U}\right)^{2},\left(y^{L}\right)^{2}+\left(y^{M}\right)^{2}+\left(y^{U}\right)^{2} .\right.
\end{aligned}
$$

According to formulas (1) and (2), it can be derived that

$$
\begin{aligned}
& r s_{\text {Max }}\left(\tilde{y}, \widetilde{z}^{*+}\right)=\frac{\left(y^{L} z^{*+L}+y^{M} z^{*+M}+y^{U} z^{*+U}\right)^{(1 / 2)}}{\left[\left(z^{*+L}\right)^{2}+\left(z^{*+M}\right)^{2}+\left(z^{*+U}\right)^{2}\right]^{(1 / 2)}}, \\
& r s_{\operatorname{Max}}\left(\tilde{x}, \widetilde{z}^{*+}\right)=\frac{\left(x^{L} z^{*+L}+x^{M} z^{*+M}+x^{U} z^{*+U}\right)^{(1 / 2)}}{\left[\left(z^{*+L}\right)^{2}+\left(z^{*+M}\right)^{2}+\left(z^{*+U}\right)^{2}\right]^{(1 / 2)}}, \\
& r s_{\text {Min }}\left(\tilde{y}, \widetilde{z}^{*-}\right)=\frac{\left[\left(z^{*-L}\right)^{2}+\left(z^{*-M}\right)^{2}+\left(z^{*-U}\right)^{2}\right]^{(1 / 2)}}{\left(y^{L} z^{*-L}+y^{M} z^{*-M}+y^{U} z^{*-U}\right)^{(1 / 2)}}, \\
& r s_{\text {Min }}\left(\tilde{x}, \widetilde{z}^{*-}\right)=\frac{\left[\left(z^{*-L}\right)^{2}+\left(z^{*-M}\right)^{2}+\left(z^{*-U}\right)^{2}\right]^{(1 / 2)}}{\left(y^{L} z^{*-L}+y^{M} z^{*-M}+y^{U} z^{*-U}\right)^{(1 / 2)}} .
\end{aligned}
$$

Therefore,

$$
\begin{aligned}
& r s_{\operatorname{Max}}\left(\tilde{y}, \tilde{z}^{*+}\right)>r s_{\operatorname{Max}}\left(\tilde{x}, \widetilde{z}^{*+}\right) \vee r s_{\operatorname{Min}}\left(\tilde{y}, \tilde{z}^{*-}\right)<r s_{\operatorname{Min}}\left(\widetilde{x}, \widetilde{z}^{*-}\right) \Longleftrightarrow \frac{z^{*+L}}{z^{*+U}} \cdot y^{L}+\frac{z^{*+M}}{z^{*+U}} \cdot y^{M} \\
& \quad+y^{U}>\frac{z^{*+L}}{z^{*+U}} \cdot x^{L}+\frac{z^{*+M}}{z^{*+U}} \cdot x^{M}+x^{U} \vee \frac{z^{*-L}}{z^{*-U}} \cdot y^{L}+\frac{z^{*-M}}{z^{*-U}} \cdot y^{M}+y^{U}>\frac{z^{*-L}}{z^{*-U}} \cdot x^{L}+\frac{z^{*-M}}{z^{*-U}} \cdot x^{M} \\
& \quad+x^{U} \Longleftrightarrow q_{1}^{*+} y^{L}+q_{2}^{*+} y^{M}+y^{U}>q_{1}^{*+} x^{L}+q_{2}^{*+} x^{M}+x^{U} \vee q_{1}^{*-} y^{L}+q_{2}^{*-} y^{M}+y^{U}>q_{1}^{*-} x^{L}+q_{2}^{*-} x^{M}+x^{U} .
\end{aligned}
$$


Thus, formulas (8) hold. Proof is completed.

We can use results in Theorem 2 to compare the dominance relation between different triangular fuzzy numbers, 2 that is, by calculating the max-relative similarity degree or min-relative similarity degree between triangular fuzzy numbers and ideal points or by comparing the sum of attribute value relations of small, special, and large elementsof triangular fuzzy numbers.

Definition 6. Let alternative decision-making objects composed of triangular fuzzy number sequence are $X=\left\{\tilde{x}_{1}, \tilde{x}_{2}, \ldots, \tilde{x}_{m}\right\}$ and $Y=\left\{\tilde{y}_{1}, \tilde{y}_{2}, \ldots, \tilde{y}_{m}\right\}$. The triangular fuzzy number-based positive and negative ideal decision-making object composed of positive and negative ideal point sequence is defined as $Z^{*+}=\left\{\widetilde{z}_{1}^{*+}, \widetilde{z}_{2}^{*+}, \ldots, \widetilde{z}_{m}^{*+}\right\}$ and $Z^{*-}=\left\{\tilde{z}_{1}^{*-}, \widetilde{z}_{2}^{*-}, \ldots, \tilde{z}_{m}^{*-}\right\}$, where $\tilde{x}_{j}=\left[x_{j}^{L}, x_{j}^{M}, x_{j}^{U}\right]$, $\tilde{y}_{j}=\left[y_{j}^{L}, y_{j}^{M}, y_{j}^{U}\right], \quad \tilde{z}_{j}^{*+}=, \quad\left[z_{j}^{*+L}, z_{j}^{*+M}, z_{j}^{*+U}\right]$, $\tilde{z}_{j}^{*-}=\left[z_{j}^{*-L}, z_{j}^{*-M}, z_{j}^{*-U}\right], j=1,2, \ldots, m$; if

$$
\begin{aligned}
& \mathrm{RS}_{\text {Max }}\left(Y, Z^{*+}\right)>\mathrm{RS}_{\mathrm{Max}}\left(X, Z^{*+}\right) \text { or } \mathrm{RS}_{\text {Min }}\left(Y, Z^{*-}\right) \\
& \quad<\mathrm{RS}_{\text {Min }}\left(X, Z^{*-}\right),
\end{aligned}
$$

then the alternative decision-making object $Y$ is called $Y$ superior to $X$, defined as $Y>X$. Obviously, the greater the maxrelative similarity degree with the positive ideal decision-making object of triangular fuzzy number is or the smaller the maxrelative similarity degree with the negative ideal decision-making object of triangular fuzzy number is, the greater the superiority of the alternative decision-making objects is.

Theorem 3. If and only if positive and negative ideal decision-making objects of triangular fuzzy number are the optimal decision-making objects,

$$
\begin{aligned}
& Y>X \Longleftrightarrow \mathrm{RS}_{\mathrm{Max}}\left(Y, Z^{*+}\right)>\mathrm{RS}_{\mathrm{Max}}\left(X, Z^{*+}\right) \vee \mathrm{RS}_{\mathrm{Min}}\left(Y, Z^{*-}\right) \\
&<\mathrm{RS}_{\mathrm{Min}}\left(X, Z^{*-}\right) \Longleftrightarrow \\
& \sum_{j=1}^{m}\left(q_{1 j}^{*+} y_{j}^{L}+q_{2 j}^{*+} y_{j}^{M}+y_{j}^{U}\right)^{(1 / 2)} \\
&> p^{*+} \sum_{j=1}^{m}\left(q_{1 j}^{*+} x_{j}^{L}+q_{2 j}^{*+} x_{j}^{M}+x_{j}^{U}\right)^{(1 / 2)} \\
& \vee \sum_{j=1}^{m}\left(q_{1 j}^{*-} y_{j}^{L}+q_{2 j}^{*-} y_{j}^{M}+y_{j}^{U}\right)^{(1 / 2)}> \\
& p^{*-} \sum_{j=1}^{m}\left(q_{1 j}^{*-} x_{j}^{L}+q_{2 j}^{*-} x_{j}^{M}+x_{j}^{U}\right)^{(1 / 2)}, \operatorname{Among} q_{1 j}^{*+}=\frac{z_{j}^{*+L}}{z_{j}^{*+U}}, \\
& q_{2 j}^{*+}=\frac{z_{j}^{*+M}}{z_{j}^{*+U}}, \\
& q_{1 j}^{*-}=\frac{z_{j}^{*-L}}{z_{j}^{*-U}}, \\
& q_{2 j}^{*-}=\frac{z_{j}^{*-M}}{z_{j}^{*-U}} \text { and } p^{*+}=\frac{\min _{1 \leq j \leq m}\left\{\left(z_{j}^{*+U}\right)^{(1 / 2)}\right\}}{\max _{1 \leq j \leq m}\left\{\left(z_{j}^{*+U}\right)^{(1 / 2)}\right\}}, \\
& p^{*-}=\frac{\min _{1 \leq j \leq m}\left\{\left(z_{j}^{*-U}\right)^{(1 / 2)}\right\}}{\max _{1 \leq j \leq m}\left\{\left(z_{j}^{*-U}\right)^{(1 / 2)} .\right.} .
\end{aligned}
$$

Proof. Obviously, according to Definition 6,

$$
\begin{aligned}
Y> & X \Longleftrightarrow \mathrm{RS}_{\text {Max }}\left(Y, Z^{*+}\right)>\mathrm{RS}_{\text {Max }}\left(X, Z^{*+}\right) \vee \operatorname{RS}_{\text {Min }}\left(Y, Z^{*-}\right) \\
& <\mathrm{RS}_{\text {Min }}\left(X, Z^{*-}\right) .
\end{aligned}
$$

$\operatorname{As} Z^{*+}=\left\{\widetilde{z}_{1}^{*+}, \widetilde{z}_{2}^{*+}, \ldots, \widetilde{z}_{m}^{*+}\right\}$

and

$Z^{*-}=\left\{\widetilde{z}_{1}^{*-}, \tilde{z}_{2}^{*-}, \ldots, \widetilde{z}_{m}^{*-}\right\}$ are, respectively, triangular fuzzy number based positive and negative ideal decision-making objects composed of positive and negative ideal points sequence, $\quad \tilde{z}_{j}^{*+}=\left[z_{j}^{*+L}, z_{j}^{*+M}, z_{j}^{*+U}\right] \quad$ and $\widetilde{z}_{j}^{*-}=\left[z_{j}^{*-L}, z_{j}^{*-M}, z_{j}^{*-U}\right]$ are triangular fuzzy numberbased positive and negative ideal points; it can be seen that

$$
\begin{aligned}
0<z_{j}^{*+L} & \leq z_{j}^{*+M} \leq z_{j}^{*+U}, 0<z_{j}^{*-L} \\
& \leq z_{j}^{*-M} \leq z_{j}^{*-U}, \quad j=1,2, \ldots, m \\
z_{j}^{*+L} & \geq \max \left\{x_{j}^{L}, y_{j}^{L}\right\}, \\
z_{j}^{*+M} & \geq \max \left\{x_{j}^{M}, y_{j}^{M}\right\}, \\
z_{j}^{*+U} & \geq \max \left\{x_{j}^{U}, y_{j}^{U}\right\}, \\
z_{j}^{*-L} & \leq \min \left\{x_{j}^{L}, y_{j}^{L}\right\}, \\
z_{j}^{*-M} & \leq \min \left\{x_{j}^{M}, y_{j}^{M}\right\}, \\
z_{j}^{*-U} & \leq \min \left\{x_{j}^{U}, y_{j}^{U}\right\}, \quad j=1,2, \ldots, m,
\end{aligned}
$$

and, therefore,

$$
\begin{aligned}
& q_{1 j}^{*+}= \frac{z_{j}^{*+L}}{z_{j}^{*+U} \leq 1,} \\
& q_{2 j}^{*+}= \frac{z_{j}^{*+M}}{z_{j}^{*+U}} \leq 1, \\
& q_{1 j}^{*-}= \frac{z_{j}^{*-L}}{z_{j}^{*-U} \leq 1,} \\
& q_{2 j}^{*-}= \frac{z_{j}^{*-M}}{z_{j}^{*-U} \leq 1, \quad j=1,2, \ldots, m} \\
&\left(z_{j}^{*+L}\right)^{2}+\left(z_{j}^{*+M}\right)^{2}+\left(z_{j}^{*+U}\right)^{2} \\
& \quad \geq \max \left\{\left(x_{j}^{L}\right)^{2}+\left(x_{j}^{M}\right)^{2}+\left(x_{j}^{U}\right)^{2},\left(y_{j}^{L}\right)^{2}+\left(y_{j}^{M}\right)^{2}+\left(y_{j}^{U}\right)^{2}\right\}, \\
&\left(z_{j}^{*-L}\right)^{2}+\left(z_{j}^{*-M}\right)^{2}+\left(z_{j}^{*-U}\right)^{2} \\
& \quad \leq \min \left\{\left(x_{j}^{L}\right)^{2}\right. \\
&\left.\quad+\left(x_{j}^{M}\right)^{2}+\left(x_{j}^{U}\right)^{2},\left(y_{j}^{L}\right)^{2}+\left(y_{j}^{M}\right)^{2}+\left(y_{j}^{U}\right)^{2}\right\}, \quad j=1,2, \ldots, m .
\end{aligned}
$$

According to formulas (3) and (4), we can get 


$$
\begin{aligned}
& \operatorname{RS}_{\text {Max }}\left(Y, Z^{*+}\right)=\frac{\sum_{j=1}^{m}\left(y_{j}^{L} z_{j}^{*+L}+y_{j}^{M} z_{j}^{*+M}+y_{j}^{U} z_{j}^{*+U}\right)^{(1 / 2)}}{\sum_{j=1}^{m}\left[\left(z_{j}^{*+L}\right)^{2}+\left(z_{j}^{*+M}\right)^{2}+\left(z_{j}^{*+U}\right)^{2}\right]^{(1 / 2)}}, \\
& \operatorname{RS}_{\text {Max }}\left(X, Z^{*+}\right)=\frac{\sum_{j=1}^{m}\left(y_{j}^{L} z_{j}^{*+L}+y_{j}^{M} z_{j}^{*+M}+y_{j}^{U} z_{j}^{*+U}\right)^{(1 / 2)}}{\sum_{j=1}^{m}\left[\left(z_{j}^{*+L}\right)^{2}+\left(z_{j}^{*+M}\right)^{2}+\left(z_{j}^{*+U}\right)^{2}\right]^{(1 / 2)}} \\
& \operatorname{RS}_{\text {Min }}\left(Y, Z^{*-}\right)=\frac{\sum_{j=1}^{m}\left[\left(z_{j}^{*-L}\right)^{2}+\left(z_{j}^{*-M}\right)^{2}+\left(z_{j}^{*-U}\right)^{2}\right]^{(1 / 2)}}{\sum_{j=1}^{m}\left(y_{j}^{L} z_{j}^{*-L}+y_{j}^{M} z_{j}^{*-M}+y_{j}^{U} z_{j}^{*-U}\right)^{(1 / 2)}},
\end{aligned}
$$

$$
\operatorname{RS}_{\operatorname{Min}}\left(X, Z^{*-}\right)=\frac{\sum_{j=1}^{m}\left[\left(z_{j}^{*-L}\right)^{2}+\left(z_{j}^{*-M}\right)^{2}+\left(z_{j}^{*-U}\right)^{2}\right]^{(1 / 2)}}{\sum_{j=1}^{m}\left(y_{j}^{L} z_{j}^{*-L}+y_{j}^{M} z_{j}^{*-M}+y_{j}^{U} z_{j}^{*-U}\right)^{(1 / 2)}} .
$$

As a result,

$$
\begin{aligned}
& \operatorname{RS}_{\text {Max }}\left(Y, Z^{*+}\right)>\operatorname{RS}_{\text {Max }}\left(X, Z^{*+}\right) \vee \mathrm{RS}_{\text {Min }}\left(Y, Z^{*-}\right)<\operatorname{RS}_{\text {Min }}\left(X, Z^{*-}\right) \\
& \Longleftrightarrow \sum_{j=1}^{m}\left(\frac{z_{j}^{*+L}}{z_{j}^{*+U}} \cdot y_{j}^{L}+\frac{z_{j}^{*+M}}{z_{j}^{*+U}} \cdot y_{j}^{M}+y_{j}^{U}\right)^{(1 / 2)}\left(z_{j}^{*+U}\right)^{(1 / 2)}>\sum_{j=1}^{m}\left(\frac{z_{j}^{*+L}}{z_{j}^{*+U}} \cdot x_{j}^{L}+\frac{z_{j}^{*+M}}{z_{j}^{*+U}} \cdot x_{j}^{M}+x_{j}^{U}\right)^{(1 / 2)}\left(z_{j}^{*+U}\right)^{(1 / 2)}
\end{aligned}
$$

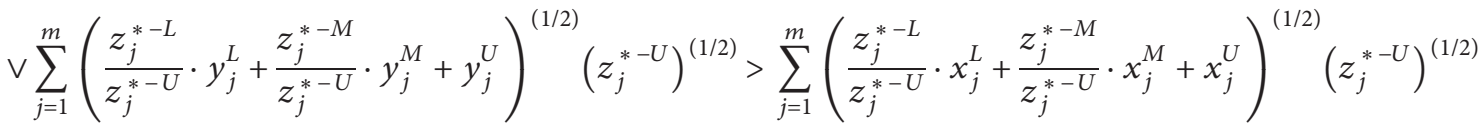

$$
\begin{aligned}
& \Longleftrightarrow \sum_{j=1}^{m}\left(\frac{z_{j}^{*+L}}{z_{j}^{*+U}} \cdot y_{j}^{L}+\frac{z_{j}^{*+M}}{z_{j}^{*+U}} \cdot y_{j}^{M}+y_{j}^{U}\right)^{(1 / 2)}>\frac{\min _{1 \leq j \leq m}\left\{\left(z_{j}^{*+U}\right)^{(1 / 2)}\right\}}{\max _{1 \leq j \leq m}\left\{\left(z_{j}^{*+U}\right)^{(1 / 2)}\right\}} \sum_{j=1}^{m}\left(\frac{z_{j}^{*+L}}{z_{j}^{*+U}} \cdot x_{j}^{L}+\frac{z_{j}^{*+M}}{z_{j}^{*+U}} \cdot x_{j}^{M}+x_{j}^{U}\right)^{(1 / 2)} \\
& \vee \sum_{j=1}^{m}\left(\frac{z_{j}^{*-L}}{z_{j}^{*-U}} \cdot y_{j}^{L}+\frac{z_{j}^{*-M}}{z_{j}^{*-U}} \cdot y_{j}^{M}+y_{j}^{U}\right)^{(1 / 2)}>\frac{\min _{1 \leq j \leq m}\left\{\left(z_{j}^{*-U}\right)^{(1 / 2)}\right\}}{\max _{1 \leq j \leq m}\left\{\left(z_{j}^{*-U}\right)^{(1 / 2)}\right\}} \sum_{j=1}^{m}\left(\frac{z_{j}^{*-L}}{z_{j}^{*-U}} \cdot x_{j}^{L}+\frac{z_{j}^{*-M}}{z_{j}^{*-U}} \cdot x_{j}^{M}+x_{j}^{U}\right)^{(1 / 2)} \\
& \Longleftrightarrow \sum_{j=1}^{m}\left(q_{1 j}^{*+} y_{j}^{L}+q_{2 j}^{*+} y_{j}^{M}+y_{j}^{U}\right)^{(1 / 2)}>p^{*+} \sum_{j=1}^{m}\left(q_{1 j}^{*+} x_{j}^{L}+q_{2 j}^{*+} x_{j}^{M}+x_{j}^{U}\right)^{(1 / 2)} \vee \sum_{j=1}^{m}\left(q_{1 j}^{*-} y_{j}^{L}+q_{2 j}^{*-} y_{j}^{M}+y_{j}^{U}\right)^{(1 / 2)} \\
& >p^{*-} \sum_{j=1}^{m}\left(q_{1 j}^{*-} x_{j}^{L}+q_{2 j}^{*-} x_{j}^{M}+x_{j}^{U}\right)^{(1 / 2)}
\end{aligned}
$$

Now, we can see that formula (15) holds, which completes the proof.

The above theoremcan be exploited to compare the advantages between alternative decision objects, by comparing the Max-relative or Min-relative similarity degree between alternative decision objects and ideal decision objects, the sum of attribute value sequence relations of triangular fuzzy number small, special, and large elements of alternative decision objects.

In the process of analyzing the advantages and disadvantages of the UMADM problem with unknown attribute weight and without any decision object preference we could assume that the set of alternative decision objects in the decision space is $\left\{X_{i} \mid i=1,2, \ldots, n\right\}$. It is generally considered that the greater the max-relative similarity degree $\mathrm{RS}_{\mathrm{Max}}\left(X_{i}, Z^{*+}\right)$ between the alternative decision-making objects $X_{i}$ and the positive ideal optimal decision-making objects is, the smaller the min-relative similarity degree $\mathrm{RS}_{\text {Min }}\left(X_{i}, Z^{*-}\right)$ between the alternative decision-making objects $X_{i}$ and the negative ideal optimal decision-making objects is, and the better the corresponding alternative decision-making object is. However, it is pointed out in [14] that the alternative decision-making objects are not necessarily close to the positive ideal optimal object and far 
away from the negative ideal optimal object at the same time. In order to obtain the optimal approach point to the positive and negative ideal decision-making objects, a concept of overall relative similarity degree $\operatorname{ORS}_{\mathrm{TFN}}\left(X_{i}\right)$ $[7,15]$ (Definition 7) is introduced to represent the degree of relative difference between an alternative decisionmaking object approaching the optimal ideal point, namely, the point close to the positive ideal object, and that far away from the negative ideal optimal object point.

Definition 7. Let the decision-making object composed of triangular fuzzy number sequence be $X_{i}=\left\{\tilde{x}_{i_{1}}, \tilde{x}_{i_{i}}, \ldots, \tilde{x}_{i_{m}}\right\}$, $i=1,2, \ldots, n$ and the positive and negative ideal decisionmaking objects composed of positive and negative ideal point sequence of triangular fuzzy number be, respectively, $Z^{*+}=\left\{\widetilde{z}_{1}^{*+}, \widetilde{z}_{2}^{*+}, \ldots, \widetilde{z}_{m}^{*+}\right\} \quad$ and $Z^{*-}=\left\{\widetilde{z}_{1}^{*-}, \widetilde{z}_{2}^{*-}, \ldots, \widetilde{z}_{m}^{*-}\right\}$; then

$$
\begin{aligned}
\operatorname{ORS}_{\mathrm{TFN}}\left(X_{i}\right)= & \frac{\mathrm{RS}_{\mathrm{Max}}\left(X_{i}, Z^{*+}\right)}{\max _{1 \leq i \leq n}\left\{\operatorname{RS}_{\mathrm{Max}}\left(X_{i}, Z^{*+}\right)\right\}} \\
& -\frac{\operatorname{RS}_{\text {Min }}\left(X_{i}, Z^{*-}\right)}{\min _{1 \leq i \leq n}\left\{\operatorname{RS}_{\mathrm{Min}}\left(X_{i}, Z^{*-}\right)\right\}},
\end{aligned}
$$

where $\operatorname{ORS}_{\mathrm{TFN}}\left(X_{i}\right)$ is the overall relative similarity degree of the comparison of Max-relative and Min- relative similarity degrees in the set of the alternative decision-making objects between the selected decision-making objects and the positive and negative ideal decision-making objects.

Theorem 4. $\operatorname{ORS}_{T F N}\left(X_{i}\right) \leq 0, i=1,2, \ldots, n$.

Proof. According to formula (21), it is easy to know

$$
\frac{\mathrm{RS}_{\mathrm{Max}}\left(X_{i}, Z^{*+}\right)}{\max _{1 \leq i \leq n}\left\{\mathrm{RS}_{\operatorname{Max}}\left(X_{i}, Z^{*+}\right)\right\}} \leq 1 \leq \frac{\mathrm{RS}_{\text {Min }}\left(X_{i}, Z^{*-}\right)}{\min _{1 \leq i \leq n}\left\{\mathrm{RS}_{\text {Min }}\left(X_{i}, Z^{*-}\right)\right\}} .
$$

We can get

$$
\frac{\mathrm{RS}_{\text {Max }}\left(X_{i}, Z^{*+}\right)}{\max _{1 \leq i \leq n}\left\{\operatorname{RS}_{\text {Max }}\left(X_{i}, Z^{*+}\right)\right\}}-\frac{\mathrm{RS}_{\text {Min }}\left(X_{i}, Z^{*-}\right)}{\min _{1 \leq i \leq n}\left\{\operatorname{RS}_{\text {Min }}\left(X_{i}, Z^{*-}\right)\right\}} \leq 0,
$$

which completes the proof.

If the alternative decision object $X_{i}^{*} \in\left\{X_{i} \mid i=1,2, \ldots, n\right\}$ also satisfies

$$
\begin{aligned}
\operatorname{RS}_{\text {Max }}\left(X_{i}^{*}, Z^{*+}\right)= & \max _{1 \leq i \leq n}\left\{\operatorname{RS}_{\text {Max }}\left(X_{i}, Z^{*+}\right)\right\} \wedge \operatorname{RS}_{\text {Min }}\left(X_{i}^{*}, Z^{*-}\right) \\
& =\min _{1 \leq i \leq n}\left\{\operatorname{RS}_{\text {Min }}\left(X_{i}, Z^{*-}\right)\right\},
\end{aligned}
$$

then $\mathrm{ORS}_{\mathrm{TFN}}\left(X_{i}^{*}\right)=0$; whichreaches the maximum. At this time, the max-relative similarity between the alternative decision-making object $X_{i}^{*}$ and the positive ideal optimal decision-making object is the largest and the most approximate, while $t$, the max-relative similarity between the alternative decision-making object $X_{i}^{*}$ and the positive ideal optimal decision-making object, is the smallest and farthest away. If $\mathrm{ORS}_{\mathrm{TFN}}\left(X_{i}\right)$ gradually decreases, the alternative decision object $X_{i}$ deviates from the positive ideal optimal object point and gets close to the negative ideal optimal object point, making it difficult to meet the requirements of decision-makers. Therefore, the overall relative similarity degree $\mathrm{ORS}_{\mathrm{TFN}}\left(X_{i}\right)$ given in this paper excels in picking and sorting the alternative decision objects $\left\{X_{i} \mid i=1,2, \ldots, n\right\}$. Alternative decision objects $X_{i_{1}}$ are better than $X_{i_{2}}$, marked as $X_{i_{1}}>X_{i_{2}}\left(i_{1}, i_{2}=1,2, \ldots, n\right)$.

\section{A Triangular Fuzzy Number-Based Decision- Making Object Relative Similarity Programming Model}

For the UMADM problem with unknown index weight and no preference for decision-making objects, we combine the relevant theory of the weighting algorithm for maximizing the comparative possibility in cooperative game theory [23] with the advantage relation theories to comparative relative similarity degree of triangular fuzzy number presented in this paper. By considering the risk attitude of decision makers, a new attribute weighting rule based on relative similar programming model of triangular fuzzy number decision-making objects is proposed from the perspective of determining the advantages and disadvantages of the alternatives $[7,15]$ : after eliminating the influence of different physical dimensions, namely, unifying the incommensurability between different attribute utility measures, if the comprehensive relative similarity between all alternative decision-making objects under the same attribute utility is smaller (i.e., the difference between attribute measure values is greater), the attribute is weighted. In the contrary, if the comprehensive relative similarity of all candidate decisionmaking objects is greater (i.e., the difference of attribute measure values is smaller) under the same attribute utility, the weighting value of attributes should be reduced accordingly. In particular, if the comprehensive relative similarity of all the candidate decision-making objects synthesized among the measure values under the same attribute utility reaches the maximum-that is, it is equal to 1 (i.e., there is no difference in attribute measure values) then the attribute weighting value can be assigned to zero accordingly. From the perspective of similarity measure principle, the comparative relative similarity value assembled between the attribute measure values of the alternative decision objects, that is, the difference degree of the attribute measure value data, is the main basis and key factor for judging the quality of the decision results. Next, the paper will establish a comparative relative similarity planning model by using the attribute utility measure value information of the decision objects and determine the optimal attribute weight vector by solving it. Then, the decision information gathered and fused under the weight of the vector can not only reduce the similarity among the alternative decision objects but also expand the difference and discrimination among the alternative decision objects, which is more conducive to the comparative judgment, and picking and sorting of the optimal decision objects. 
In the process of judging the merits and demerits of decision objects for a UMADM problem, it is assumed that all alternative decision-making objects $X_{i}$ in the utility measurement of each attribute $u_{j}$ will obtain a matrix $\widetilde{X}=$ $\left(\tilde{x}_{i j}\right)_{n \times m}$ composed of the measured values of the attributes of all the initial decision objects about $u_{j}$, which is called the initial triangular fuzzy number decision matrix. Here, $\tilde{x}_{i j}=\left[x_{i j}^{L}, x_{i j}^{M}, x_{i j}^{U}\right] \quad$ where $\quad M=\{1,2, \ldots, m\}$, $N=\{1,2, \ldots, n\}$. Let $I_{j}(j=1,2)$ denote the subscript set of the most common benefit attributes and cost benefit. It is easy to see $M=I_{1} \cup I_{2}$. In order to fuse the incommensurability and contradiction between the utility measure data of different attributes and eliminate the influence of different physical dimensions on the judgment and ranking results of decision objects, we adopt the following formulas to transform the initial triangular fuzzy number decision matrix $\widetilde{X}$ into the standard triangular fuzzy number decision matrix $\widetilde{R}=\left(\widetilde{r}_{i j}\right)_{n \times m}[7,15]$ :

$$
\begin{aligned}
& \tilde{r}_{i j}=\frac{\tilde{x}_{i j}}{\left\|\tilde{x}_{j}\right\|}, \quad i \in N, j \in I_{1}, \\
& \tilde{r}_{i j}=\frac{\left(1 / \widetilde{x}_{i j}\right)}{\left\|\left(1 / \tilde{x}_{j}\right)\right\|}, \quad i \in N, j \in I_{2},
\end{aligned}
$$

where $\tilde{r}_{i j}=\left[r_{i j}^{L}, r_{i j}^{M}, r_{i j}^{U}\right]$ is a normalized triangular fuzzy number and $\|\cdot\|$ is norm of a vector, $\left\|\tilde{x}_{j}\right\|=\sqrt{\sum_{i=1}^{n} \tilde{x}_{i j}^{2}}$, $\operatorname{and}\left\|\left(1 / \widetilde{x}_{j}\right)\right\|=\sqrt{\sum_{i=1}^{n}\left(1 / \tilde{x}_{i j}\right)^{2}}$. According to the operation relation rule of triangular fuzzy numbers, formulas (25) and (26) can be changed into the following formulas:

$$
\begin{aligned}
& \left\{\begin{array}{l}
r_{i j}^{L}=\frac{x_{i j}^{L}}{\sqrt{\sum_{i=1}^{n}\left(x_{i j}^{U}\right)^{2}}}, \\
r_{i j}^{M}=\frac{x_{i j}^{M}}{\sqrt{\sum_{i=1}^{n}\left(x_{i j}^{M}\right)^{2}}}, \quad i \in N, j \in I_{1}, \\
r_{i j}^{U}=\frac{x_{i j}^{U}}{\sqrt{\sum_{i=1}^{n}\left(x_{i j}^{L}\right)^{2}}}, \\
r_{i j}^{L}=\frac{x_{i j}^{L}}{\sqrt{\sum_{i=1}^{n}\left(x_{i j}^{U}\right)^{2}}}, \\
r_{i j}^{M}=\frac{x_{i j}^{M}}{\sqrt{\sum_{i=1}^{n}\left(x_{i j}^{M}\right)^{2}}}, \quad i \in N, j \in I_{2} . \\
r_{i j}^{U}=\frac{x_{i j}^{U}}{\sqrt{\sum_{i=1}^{n}\left(x_{i j}^{L}\right)^{2}}},
\end{array}\right.
\end{aligned}
$$

Therefore, for the $j$ th attribute, the total comprehensive relative similarity degree between the alternative decision objects and other alternative decision objects is

$$
r s\left(u_{j}\right)=\sum_{i=1}^{n} r s_{i}\left(u_{j}\right)=\sum_{i=1}^{n} \sum_{k=1, k \neq i}^{n} r s_{\mathrm{TFN}}\left(\widetilde{r}_{i j}, \widetilde{r}_{k j}\right), \quad i, k \in N, j \in M .
$$

For a UMADM problem whose attribute weight information is completely unknown, we can assume its attribute weight vector is expressed by $\mathbf{W}=\left(w_{1}, w_{2}, \ldots, w_{m}\right)$, $0 \leq w_{j} \leq 1, j \in M$, and satisfyingthe constraints $\sum_{j=1}^{m} w_{j}^{2}=1$.

According to the attribute weighting rules based on decision-making object relative similarity programming model proposed in this paper, under the consideration of the risk awareness of decision-makers and unknown attribute weights and no preference for decision objects, the determination of the optimal solution of weighting vectors $\mathbf{W}$ should make the weighted sum of the total comprehensive relative similarity of all attributes to all alternative decision objects minimum under the action of weighting vectors $\mathbf{W}$. In other words, the optimal solution of the weighting vector $\mathbf{W}$ should be determined so that the weighted sum of the reciprocal of the total comprehensive relative similarity of all attributes to all alternative decision objects must be the largest under the action of the weighting vector $\mathbf{W}$. In order to obtain the optimal weight vector $\mathbf{W}$, we can construct the triangular fuzzy number-based decision-making object relative similarity programming model of UMADM (TFNDMORSPM):

$$
\begin{aligned}
& \max \varphi(\mathbf{W})=\sum_{j=1}^{m} \frac{1}{r s\left(u_{j}\right)} \cdot w_{j}=\sum_{j=1}^{m} \frac{1}{\sum_{i=1}^{n} \sum_{k=1, k \neq i}^{n} r s_{\mathrm{TFN}}\left(\widetilde{r}_{i j}, \widetilde{r}_{k j}\right)} \cdot w_{j}, \\
& \text { s.t. } \quad \sum_{j=1}^{m} w_{j}^{2}=1, w_{j} \geq 0, i, k \in N, j \in M .
\end{aligned}
$$

By solving the optimization model, the optimal solution 


$$
w_{j}^{*}=\frac{\left(1 / \sum_{i=1}^{n} \sum_{k=1, k \neq i}^{n} r s_{\mathrm{TFN}}\left(\widetilde{r}_{i j}, \widetilde{r}_{k j}\right)\right)}{\sqrt{\sum_{j=1}^{m}\left(1 /\left[\sum_{i=1}^{n} \sum_{k=1, k \neq i}^{n} r s_{\mathrm{TFN}}\left(\widetilde{r}_{i j}, \widetilde{r}_{k j}\right)\right]^{2}\right)}}, \quad i, k \in N, j \in M .
$$

In order to keep consistent with the traditional normalization usage, we will normalize the unit weight vector $w_{j}^{*}$ letting $w_{j}=\left(w_{j}^{*} / \sum_{j=1}^{m} w_{j}^{*}\right) j \in M$ and then we can get

$$
w_{j}=\frac{\left(1 / \sum_{i=1}^{n} \sum_{k=1, k \neq i}^{n} r s_{\mathrm{TFN}}\left(\widetilde{r}_{i j}, \widetilde{r}_{k j}\right)\right)}{\sum_{j=1}^{m}\left(1 / \sum_{i=1}^{n} \sum_{k=1, k \neq i}^{n} r s_{\mathrm{TFN}}\left(\widetilde{r}_{i j}, \widetilde{r}_{k j}\right)\right)}, \quad i, k \in N, j \in M .
$$

According to formula (33), it is easy to know that under the same attribute measure; the sum of the comprehensive relative similarity values compared among all the candidate decision objects is inversely proportional to the size of the attribute weight value.

Remark 1. The values of comprehensive relative similarity degree $r s_{\mathrm{TFN}}\left(\widetilde{r}_{i j}, \widetilde{r}_{k j}\right)$ in formulas (29) (33) are as follows:

$$
r s_{\mathrm{TFN}}\left(\widetilde{r}_{i j}, \widetilde{r}_{k j}\right)=\lambda r s_{\mathrm{Max}}\left(\widetilde{r}_{i j}, \widetilde{r}_{k j}\right)+(1-\lambda) r s_{\mathrm{Min}}\left(\widetilde{r}_{i j}, \widetilde{r}_{k j}\right) .
$$

The choice of $\lambda$ value depends on the risk attitude of decision-makers: when $\lambda>0.5$, decision-makers tend to avoid risks; when $\lambda=0.5$, decision-makers tend to be riskneutral; when $\lambda<0.5$, decision-makers tend to risk preference. In particular, when $\lambda=1, r s_{\mathrm{TFN}}\left(\widetilde{r}_{i j}, \widetilde{r}_{k j}\right)=r s_{\mathrm{Max}}$ $\left(\widetilde{r}_{i j}, \widetilde{r}_{k j}\right), r s_{\mathrm{TFN}}\left(\widetilde{r}_{i j}, \widetilde{r}_{k j}\right)$ is conservative max-relative similarity degree; when $\lambda=0, r s_{\mathrm{TFN}}\left(\widetilde{r}_{i j}, \widetilde{r}_{k j}\right)=\operatorname{rs} s_{\text {Min }}\left(\widetilde{r}_{i j}, \widetilde{r}_{k j}\right)$, $r s_{\mathrm{TFN}}\left(\widetilde{r}_{i j}, \widetilde{r}_{k j}\right)$ is adventurous min-relative similarity degree.

\section{Implementation Steps and Examples of the Model}

The implementation steps of UMADM's triangular fuzzy number-based decision-making objects relative similarity programming model (TFN-DMORSPM) given in this paper are as follows.

Step 1. To fuse the incommensurability and contradiction between the utility measure data of different attributes which eliminates the influence of different physical dimensions on the decision-making object's judgment and ranking results, the initial triangular fuzzy number type decision matrix $\widetilde{X}$ is converted into a normalized triangular fuzzy number type decision matrix $\widetilde{R}=\left(\widetilde{r}_{i j}\right)_{n \times m}$ according to formulas (27) and (28), where $\tilde{r}_{i j}=\left[r_{i j}^{L}, r_{i j}^{M}, r_{i j}^{U}\right]$ is a normalized triangular fuzzy number.

Step 2. Analyze and measure the normalized triangular fuzzy number based decision-making matrix $\widetilde{R}=\left(\widetilde{r}_{i j}\right)_{n \times m}$ which can reflect the attribute eigenvalue information of all alternative decision-making objects and calculate the max-relative similarity degree and the min-relative similarity degree between the attribute values of each decision-making object by using formulas (1) and (2). According to the constructed TFN-DMORSPM, the attribute weight measurement value $\mathbf{W}$ is obtained by calculating formulas (29) (34) and by applyingthe normalized triangular fuzzy number-based decision-making matrix $\widetilde{R}$ inStep 1, we can get the constructed matrix

$$
\widetilde{R}(\mathbf{W})=\left(\widetilde{r}_{i j} \cdot w_{j}\right)_{n \times m},
$$

which is the weighted normalized triangular fuzzy number decision-making matrix.

Step 3. According to the weighted normal triangular fuzzy number type decision-making matrix $\widetilde{R}(\mathbf{W})$ obtained in Step 2, the triangular fuzzy number-based positive and negative ideal decision-making objects $Z^{*+}=\left\{\widetilde{z}_{1}^{*+}, \widetilde{z}_{2}^{*+}, \ldots, \widetilde{z}_{m}^{*+}\right\} \quad$ and $\quad Z^{*-}=\left\{\widetilde{z}_{1}^{*-}, \widetilde{z}_{2}^{*-}, \ldots\right.$, $\left.\tilde{z}_{m}^{*-}\right\}$ composed of positive and negative ideal point sequences can be obtained by using formulas (5) and (6).

Step 4.Calculate all the max-relative similarity degree $\operatorname{RS}_{\mathrm{Max}}^{w}\left(X_{i}, Z^{*+}\right)$ and min-relative similarity degree $\mathrm{RS}_{\text {Min }}^{w}\left(X_{i}, Z^{*-}\right)$ of alternative decision-making objects and triangular fuzzy number-based positive and negative ideal decision-making objects by using formulas (3) and (4).

Step 5. Using formula (21) to calculate the overall relative similarity degree $\mathrm{ORS}_{\mathrm{TFN}}\left(X_{i}\right)(i=1,2, \ldots, n)$ by all the max-relative similarity and min-relative similarity based positive and negative ideal decisionmaking objects $Z^{*+}, Z^{*-}$ and alternative decision making objects $X_{i}$ in the alternative set.

Step 6.According to the overall relative similarity degree value $\operatorname{ORS}_{\mathrm{TFN}}\left(X_{i}\right)$, pick over and sort the set of the alternative decision-making objects $\left\{X_{i} \mid i=1,2, \ldots, n\right\}$ in descending order.

Example 1. In order to illustrate the practicability and effectiveness of TFN-DMORSPM, in this paper we uses the example of scheduling problem about carrier-based aircraft models in literature [34] to analyze. The performance parameters affecting carrier-based aircraft models, i.e. maximum speed $\left(u_{1}\right)$, free voyage across the sea $\left(u_{2}\right)$, maximum net load $\left(u_{3}\right)$, purchase cost $\left(u_{4}\right)$, reliability $\left(u_{5}\right)$, and maneuverability $\left(u_{6}\right)$, We consider that there are four types of aircraft $X_{i}(i=1,2, \ldots, 4)$ to choose. It is assumed that after standardized statistical processing, the utility measure values of performance parameters of various models under various indexes (attributes) are expressed in the form of triangular fuzzy numbers (all the given data is benefit data), as shown in Table 1.

Step 1. In order to unify the incommensurability and contradiction of different physical dimensions among the utility measures of initial attributes, we assume that the normalized triangular fuzzy number decision matrix $\widetilde{R}=\left(\widetilde{r}_{i j}\right)_{n \times m}$ obtained by standardized statistical processing of (27) and (28), $\widetilde{R}=\left(\widetilde{r}_{i j}\right)_{n \times m}$ is shown in Table 1. 
TABLE 1: Standardized decision information [34].

\begin{tabular}{lcccccc}
\hline Candidate aircraft model & $u_{1}$ & $u_{2}$ & $u_{3}$ & $u_{4}$ & $u_{5}$ & $u_{6}$ \\
\hline$X_{1}$ & {$[0.78,0.80,0.85]$} & {$[0.50,0.55,0.58]$} & {$[0.90,0.95,0.95]$} & {$[0.80,0.82,0.85]$} & {$[0.45,0.50,0.57]$} & {$[0.90,0.95,0.97]$} \\
$X_{2}$ & {$[0.92,0.95,1.00]$} & {$[0.95,0.97,1.00]$} & {$[0.85,0.86,0.88]$} & {$[0.65,0.69,0.71]$} & {$[0.17,0.20,0.23]$} & {$[0.47,0.51,0.55]$} \\
$X_{3}$ & {$[0.70,0.72,0.78]$} & {$[0.72,0.74,0.75]$} & {$[0.95,0.98,1.00]$} & {$[0.94,0.97,1.00]$} & {$[0.80,0.83,0.85]$} & {$[0.80,0.82,0.85]$} \\
$X_{4}$ & {$[0.85,0.88,0.90]$} & {$[0.65,0.67,0.70]$} & {$[0.90,0.95,0.96]$} & {$[0.85,0.90,0.93]$} & {$[0.46,0.50,0.52]$} & {$[0.48,0.50,0.52]$} \\
\hline
\end{tabular}

Step 2. According to the standard triangle fuzzy number decision matrix $\widetilde{R}$ composed of attribute utility measure value data in Table 1 , the max-relative similarity and min-relative similarity is obtained by (1) and (2), and then the weight measurement value of attributes $\mathbf{W}$ is calculated by formulas (29)-(34) according to TFN-DMORSPM for simplicity, we let $\lambda=0.5$; the decision-makers tend to be risk neutral) as follows:

$$
\begin{aligned}
& w_{1}=0.1542, \\
& w_{2}=0.1667, \\
& w_{3}=0.1480, \\
& w_{4}=0.1575, \\
& w_{5}=0.2000, \\
& w_{6}=0.1736 .
\end{aligned}
$$

The weighted normalized triangular fuzzy number decision matrix $\widetilde{R}(\mathbf{W})$ is constructed by using (34), and the weighted normalized decision information is obtained as shown in Table 2.

Step 3. According to the attribute value data $\widetilde{R}(\mathbf{w})$ in Table 2, the triangle fuzzy number-based positive and negative ideal decision-making objects composed of positive and negative ideal point sequences $\left(Z^{*+}\right.$ and $Z^{*-}$ ) are obtained by using formulas (5) and (6) as follows:

$$
\begin{aligned}
& Z^{*+}=\left\{\begin{array}{c}
{[1.419,1.465,1.542],[1.584,1.617,1.667],[1.406,1.450,1.480],[1.480,1.528,1.575],} \\
{[1.600,1.660,1.700],[1.562,1.649,1.684]}
\end{array}\right\} \times 10^{-1}, \\
& Z^{*-}=\left\{\begin{array}{c}
{[1.080,1.111,1.203],[0.833,0.917,0.967],[1.258,1.273,1.302],[1.024,1.087,1.118],} \\
{[0.340,0.400,0.460],[0.816,0.868,0.903]}
\end{array}\right\} \times 10^{-1} .
\end{aligned}
$$

Step 4. The max-relative similarity degree $\operatorname{RS}_{\mathrm{Max}}^{w}\left(X_{i}, Z^{*+}\right)$ and min-relative similarity degree $\mathrm{RS}_{\text {Min }}^{w}\left(X_{i}, Z^{*-}\right)$ of all the alternative decision objects $X_{i},(i=1,2, \ldots, 4)$ and the triangular fuzzy numberbased positive and negative ideal decision-making objects $\left(Z^{*+}\right.$ and $\left.Z^{*-}\right)$ are obtained by using formulas (3) and (4) as follows:

$$
\begin{aligned}
& \operatorname{RS}_{\text {Max }}^{w}\left(X_{1}, Z^{*+}\right)=0.8891, \\
& \operatorname{RS}_{\text {Max }}^{w}\left(X_{2}, Z^{*+}\right)=0.8290, \\
& \operatorname{RS}_{\text {Max }}^{\mathrm{w}}\left(X_{3}, Z^{*+}\right)=0.9466, \\
& \operatorname{RS}_{\text {Max }}^{\mathrm{w}}\left(X_{4}, Z^{*+}\right)=0.8669, \\
& \operatorname{RS}_{\text {Min }}^{w}\left(X_{1}, Z^{*-}\right)=0.8796, \\
& \operatorname{RS}_{\text {Min }}^{w}\left(X_{2}, Z^{*-}\right)=0.9221, \\
& \operatorname{RS}_{\text {Min }}^{w}\left(X_{3}, Z^{*-}\right)=0.8380, \\
& \operatorname{RS}_{\text {Min }}^{w}\left(X_{4}, Z^{*-}\right)=0.8978 .
\end{aligned}
$$

Step 5. Use formula (21) to find out the overall relative similarity degree $\operatorname{ORS}_{\mathrm{TFN}}\left(X_{i}\right),(i=1,2, \ldots, 4)$ calculated by all the max-relative similarity and min-relative similarity based positive and negative ideal decisionmaking objects $Z^{*+}$ and $Z^{*-}$ and alternative decisionmaking objects $X_{i}$ in the alternative set and then we have

$$
\begin{aligned}
& \operatorname{ORS}_{\mathrm{TFN}}\left(X_{1}\right)=-0.110, \\
& \operatorname{ORS}_{\mathrm{TFN}}\left(X_{2}\right)=-0.225, \\
& \operatorname{ORS}_{\mathrm{TFN}}\left(X_{3}\right)=0, \\
& \operatorname{ORS}_{\mathrm{TFN}}\left(X_{4}\right)=-0.156 .
\end{aligned}
$$

Step 6. According to the overall relative similarity degree value $\mathrm{ORS}_{\mathrm{TFN}}\left(X_{i}\right)$, one can pick over and sort the set of alternative decision-making objects $\left\{X_{i} \mid i=1,2, \ldots, 4\right\}$ in descending order, The result is $X_{3}>X_{1}>X_{4}>X_{2}$, and therefore $X_{3}$ which is the optimal type of carrier-based aircraft, This result is consistent with that of [34].

According to the results of Step 4- 5, it is easy to draw the geometrical comparison diagrams of max-relative similarity degree $\mathrm{RS}_{\mathrm{Max}}^{w}\left(X_{i}, Z^{*+}\right)$, min-relative similarity degree $\operatorname{RS}_{\text {Min }}^{w}\left(X_{i}, Z^{*-}\right)$, and overall relative similarity degree $\mathrm{ORS}_{\mathrm{TFN}}\left(X_{i}\right)$, as shown in Figure 1. Although the three relative similarity degree values are different, the 
TABLE 2: Weighted normalized decision information $\left(\times 10^{-1}\right)$.

\begin{tabular}{|c|c|c|c|c|c|c|}
\hline Candidate aircraft model & $u_{1}$ & $u_{2}$ & $u_{3}$ & $u_{4}$ & $u_{5}$ & $u_{6}$ \\
\hline$X_{1}$ & $\begin{array}{c}{[1.203,1.234} \\
1.311]\end{array}$ & $\begin{array}{c}{[0.833,0.917} \\
0.967]\end{array}$ & $\begin{array}{c}{[1.332,1.406} \\
1.406]\end{array}$ & $\begin{array}{c}{[1.260,1.291} \\
1.339]\end{array}$ & $\begin{array}{c}{[0.900,1.000} \\
1.140]\end{array}$ & {$[1.562,1.649,1.684$} \\
\hline$X_{2}$ & $\begin{array}{c}{[1.419,1.465} \\
1.542]\end{array}$ & $\begin{array}{c}{[1.584,1.617} \\
1.667]\end{array}$ & $\begin{array}{c}{[1.258,1.273} \\
1.302]\end{array}$ & $\begin{array}{c}{[1.024,1.087} \\
1.118]\end{array}$ & $\begin{array}{c}{[0.340,0.400} \\
0.460]\end{array}$ & {$[0.816,0.885,0.955]$} \\
\hline$X_{3}$ & $\begin{array}{c}{[1.080,1.111} \\
1.203]\end{array}$ & $\begin{array}{c}{[1.200,1.234,} \\
1.250]\end{array}$ & $\begin{array}{c}{[1.406,1.450} \\
1.480]\end{array}$ & $\begin{array}{c}{[1.480,1.528} \\
1.575]\end{array}$ & $\begin{array}{c}{[1.600,1.660} \\
1.700]\end{array}$ & {$[1.389,1.423,1.476]$} \\
\hline$X_{4}$ & $\begin{array}{c}{[1.311,1.357} \\
1.388]\end{array}$ & $\begin{array}{c}{[1.083,1.117,} \\
1.167]\end{array}$ & $\begin{array}{c}{[1.332,1.406} \\
1.421]\end{array}$ & $\begin{array}{c}{[1.339,1.417} \\
1.465]\end{array}$ & $\begin{array}{c}{[0.920,1.000} \\
1.040]\end{array}$ & {$[0.833,0.868,0.903]$} \\
\hline
\end{tabular}

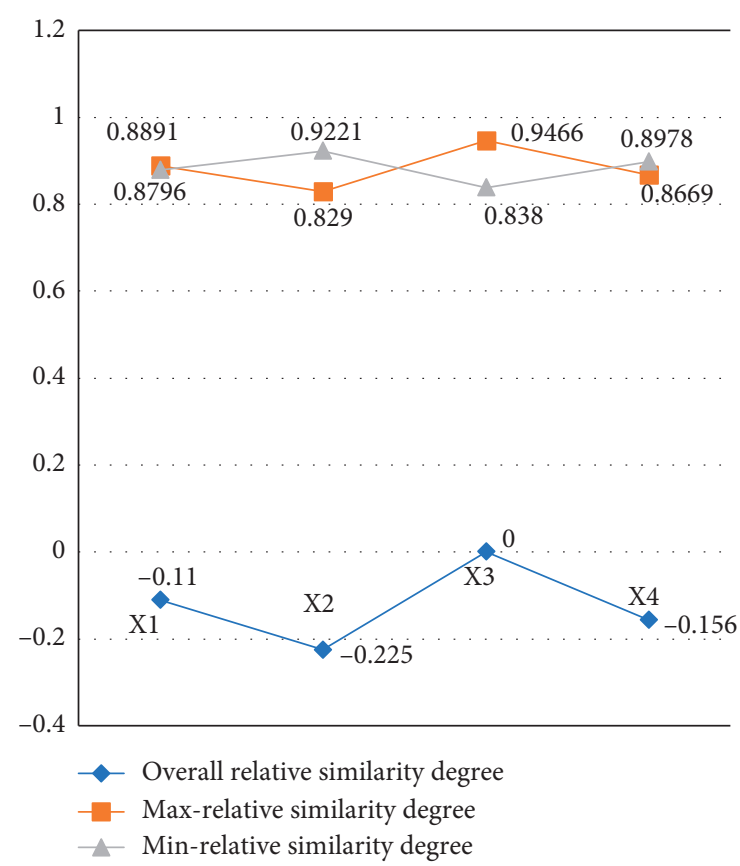

Figure 1: Geometrical comparison of the max-relative, min-relative, and overall relative similarity degree.

results of selection and ranking of the set of the alternative decision-making objects $\left\{X_{i} \mid i=1,2, \ldots, 4\right\}$ are consistent, that is $X_{3}>X_{1}>X_{4}>X_{2}$. It can be seen from Figure 1 that the max-relative similarity degree curve and the min-relative similarity degree curve present the opposite trend while the max-relative similarity degree curve and the overall relative similarity degree curve show the same trend. This is due to the fact that the positive and negative idealpoint sequences constitute different ideal decision-making objects. And the overall relative similarity degree curve appears to be steeper than the others (because the overall relative similarity degree curve aggregates the information of max-relative similarity degree and min-relative similarity degree), which can increase the discrimination.

In addition to the method of overall relative similarity advantage relation which is used to pick over and sort the alternative decision-making objects, the conclusion of Theorem 3 in this paper can also be used to sort alternative decision objects by comparing the positive and negative sequence relations sum of the weighted attribute values of small elements, special elements, and large elements of triangular fuzzy numbers of the alternative decision-making objects. We can easily obtain the following results by using (17):

$$
\begin{aligned}
& q_{11}^{*+}=0.9200, \\
& q_{21}^{*+}=0.9500, \\
& q_{12}^{*+}=0.9500, \\
& q_{22}^{*+}=0.9700, \\
& q_{13}^{*+}=0.9500, \\
& q_{23}^{*+}=0.9800, \\
& q_{14}^{*+}=0.9400, \\
& q_{24}^{*+}=0.9700, \\
& q_{15}^{*+}=0.9412, \\
& q_{25}^{*+}=0.9765, \\
& q_{16}^{*+}=0.9278, \\
& q_{26}^{*+}=0.9794, \\
& p^{*+}=0.9330, \\
& q_{11}^{*-}=0.8974, \\
& q_{21}^{*-}=0.9231, \\
& q_{12}^{*-}=0.8621, \\
& q_{22}^{*-}=0.9483, \\
& q_{13}^{*-}=0.9659, \\
& q_{23}^{*-}=0.9773, \\
& q_{14}^{*-}=0.9155, \\
& q_{24}^{*-}=0.9718, \\
& q_{15}^{*-}=0.7391, \\
& q_{25}^{*-}=0.8696, \\
& q_{16}^{*-}=0.9038, \\
& q_{26}^{*-}=0.9615,
\end{aligned}
$$


The sum of positive sequence relations $\sum_{j=1}^{6}\left(q_{1 j}^{*+} x_{i u_{j}}^{w L}+q_{2 j}^{*+} x_{i u_{j}}^{w M}+x_{i u_{j}}^{w U}\right)^{(1 / 2)}$ of the weighted attribute values is

$$
\begin{aligned}
& \sum_{j=1}^{6}\left(q_{1 j}^{*+} x_{1 u_{j}}^{w L}+q_{2 j}^{*+} x_{1 u_{j}}^{w M}+x_{1 u_{j}}^{w U}\right)^{(1 / 2)}=3.5980 \\
& \sum_{j=1}^{6}\left(q_{1 j}^{*+} x_{2 u_{j}}^{w L}+q_{2 j}^{*+} x_{2 u_{j}}^{w M}+x_{2 u_{j}}^{w U}\right)^{(1 / 2)}=3.3613 \\
& \sum_{j=1}^{6}\left(q_{1 j}^{*+} x_{3 u_{j}}^{w L}+q_{2 j}^{*+} x_{3 u_{j}}^{w M}+x_{3 u_{j}}^{w U}\right)^{(1 / 2)}=3.8266 \\
& \sum_{j=1}^{6}\left(q_{1 j}^{*+} x_{4 u_{j}}^{w L}+q_{2 j}^{*+} x_{4 u_{j}}^{w M}+x_{4 u_{j}}^{w U}\right)^{(1 / 2)}=3.5129 .
\end{aligned}
$$

The sumof negative sequence relations $\sum_{j=1}^{6}\left(q_{1 j}^{*-} x_{i u_{j}}^{w L}+q_{2 j}^{*-} x_{i u_{j}}^{w M}+x_{i u_{j}}^{w U}\right)^{(1 / 2)}$ of the weighted attribute values is

$$
\begin{aligned}
\sum_{j=1}^{6}\left(q_{1 j}^{*-} x_{1 u_{j}}^{w L}+q_{2 j}^{*-} x_{1 u_{j}}^{w M}+x_{1 u_{j}}^{w U}\right)^{(1 / 2)} & =3.5508, \\
\sum_{j=1}^{6}\left(q_{1 j}^{*-} x_{2 u_{j}}^{w L}+q_{2 j}^{*-} x_{2 u_{j}}^{w M}+x_{2 u_{j}}^{w U}\right)^{(1 / 2)} & =3.3221, \\
\sum_{j=1}^{6}\left(q_{1 j}^{*-} x_{3 u_{j}}^{w L}+q_{2 j}^{*-} x_{3 u_{j}}^{w M}+x_{3 u_{j}}^{w U}\right) & =3.7679, \\
\sum_{j=1}^{6}\left(q_{1 j}^{*-} x_{4 u_{j}}^{w L}+q_{2 j}^{*-} x_{4 u_{j}}^{w M}+x_{4 u_{j}}^{w U}\right) & =3.4645 .
\end{aligned}
$$

According to the above results, it is easy to draw the geometrical comparison diagram of the positive sequence relations sum of the weighted attribute values $\sum_{j=1}^{6}\left(q_{1 j}^{*+} x_{i u_{j}}^{w L}+q_{2 j}^{*+} x_{i u_{j}}^{w M}+x_{i u_{j}}^{w U}\right)^{(1 / 2)}$, the negative sequence relations sum of the weighted attribute values $\sum_{j=1}^{6}\left(q_{1 j}^{*-} x_{i u_{j}}^{w L}+q_{2 j}^{*-} x_{i u_{j}}^{w M}+x_{i u_{j}}^{w U}\right)^{(1 / 2)}$, and the overall relative similarity degree $\mathrm{ORS}_{\mathrm{TFN}}\left(X_{i}\right)$ of the triangular fuzzy number small element, special element and large element of the alternative decision-making objects as shown in Figure 2. Although the two sequence relations' sum of the weighted attribute values and the overall relative similarity degree is different, the results of selection and ranking of the set of the alternative decision-making objects $\quad\left\{X_{i} \mid i=1,2, \ldots, 4\right\} \quad$ are consistent, i.e., $X_{3}>X_{1}>X_{4}>X_{2}$. It can be seen from Figure 2 that the three sum still show the same trend, and the curve of positive sequence relations of the weighted attribute values and the sum of negative sequence relations of the weighted attribute values almost coincide (this is caused by the conclusion of Theorem 3 ).

Therefore, the selection and ranking of the set of the alternative decision-making objects $\left\{X_{i} \mid i=1,2, \ldots, 4\right\}$ are $X_{3}>X_{1}>X_{4}>X_{2}$, which indicates that $X_{3}$, which are still the optimal model of carrier aircraft.

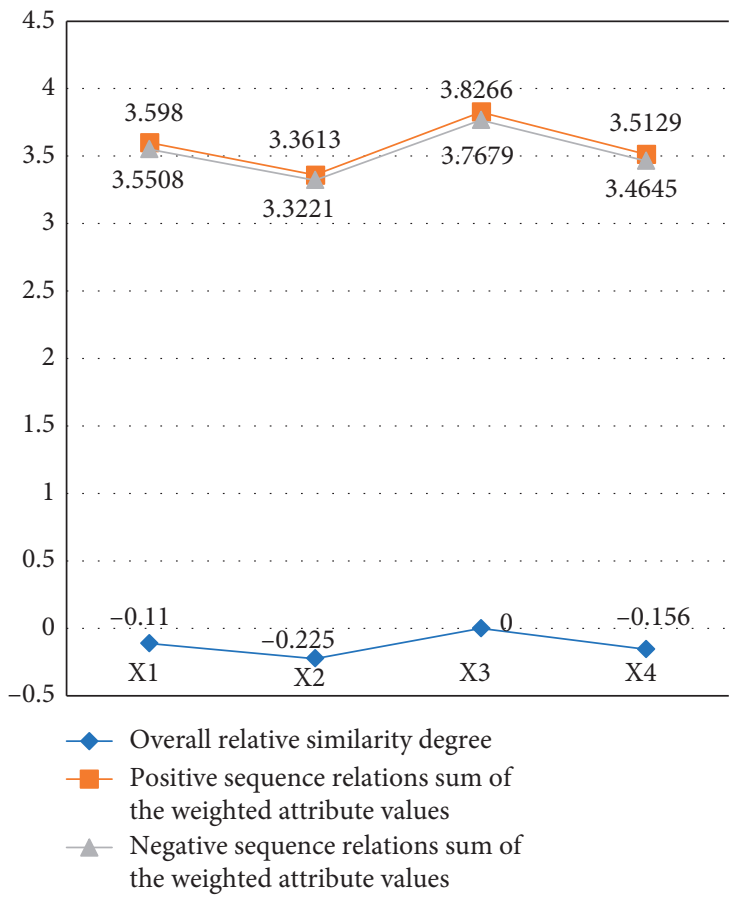

FIgURE 2: Geometrical comparison of the positive and negative sequence relations sum of the weighted attribute values and overall relative similarity degree.

In order to pick over and sort the set of alternative decision-making objects, we calculate the overall relative similarity degree value $\operatorname{ORS}_{\text {IN }}\left(X_{i}\right)$ in the set of the alternative decision-making objects and the relevant conclusions of Theorem 3 to determine the order of the alternative decision objects by using the relative similarity between the alternative decision making-objects and the optimal ideal decision-making objects. The model is simple to implement, but it cannot measure the specific value of the comparative advantage between any two decision-making objects.

In addition, we adopt the weighted multiattribute decision-making algorithms based on the triangular fuzzy number-based decision-making object maximizing deviation programming model (TFN-DMOMDPM) in [1] and the triangular fuzzy number-based decisionmaking object possibility degree relation model (TFNDMOPDRM) in [32] to make a comparative analysis on the cases of judging the advantages and disadvantages of carrier based aircraft types in [34]. After unifying the different physical dimension information between the attribute values data (the utility measure value of the performance parameters of the carrier aircraft under various indexes) of decision-making objects in UMADM problem, the attribute weight measurement formulas based on TFN-DMOMDPM [1] and TFN-DMOPDRM [32] algorithm are as follows.

(1) Calculate the attribute weight measurement formulas based on TFN-DMOMDPM 


$$
w_{j}=\frac{\sum_{i=1}^{n} \sum_{k=1}^{n} d_{\mathrm{TFN}}\left(\widetilde{r}_{i j}, \widetilde{r}_{k j}\right)}{\sum_{j=1}^{m} \sum_{i=1}^{n} \sum_{k=1}^{n} d_{\mathrm{TFN}}\left(\widetilde{r}_{i j}, \widetilde{r}_{k j}\right)}, \quad i, k \in N, j \in M .
$$

(2) Calculate the attribute weight measurement formulas based on TFN-DMOPDRM

$$
w_{j}=\frac{\sum_{k=1, k \neq j}^{m} \sum_{i=1}^{n} p\left(\widetilde{r}_{i j} \geq \widetilde{r}_{i k}\right)}{\sum_{j=1}^{m} \sum_{k=1, k \neq j}^{m} \sum_{i=1}^{n} p\left(\tilde{r}_{i j} \geq \widetilde{r}_{i k}\right)}, \quad i \in N, j, k \in M .
$$

According to the implementation steps of multiattribute decision-making algorithm of TFNDMOMDPM and TFN-DMOPDRM proposed in $[1,32]$ in dealing with UMADM problems, one has the following

(1) The result of implementation based on TFNDMOMDPM is

$$
X_{31.0000}^{\succ} X_{1} \underset{0.9890}{\succ} X_{4} \underset{0.8471}{\succ} X_{2} \circ \text {. }
$$

(2) The result of implementation based on TFNDMOPDRM is

$$
X_{3} \underset{1.0000}{\succ} X_{1} \underset{0.8382}{\succ} X_{4} \underset{0.9506}{\succ} X_{2}
$$

Therefore, $X_{3}$ is the best carrier aircraft type. The results are consistent with the results given in the paper. According to the above results, it is easy to draw the geometrical comparison diagrams of TFN-DMOMDPM, TFNDMOPDRM, and TFN-DMORSP given in the paper, as shown in Figure 3.

Although the three models to determine the weighted attribute measure formula are different, the results of selection and ranking of the set of the alternative decision-making objects $\left\{X_{i} \mid i=1,2, \ldots, 4\right\}$ are consistent, i.e., $X_{3}>X_{1}>X_{4}>X_{2}$. From Figure 3, we can see that the three curves present the same trend, and the TFN-DMOMDPM curve almost coincides with the TFN-DMOPDRM curve and is steeper than the TFNDMORSPM curve. Although it is convenient to increase the discrimination of decision, it also increases the error of decision information in the process of aggregation.

The example analysis of the carrier aircraft types shows that different attribute weight vectors can be obtained by using the attribute weighting algorithm based on triangular fuzzy numberbased decision-making object relative similarity programming model (TFN-DMORSPM) proposed in the paper and the attribute weighting algorithm based on TFN-DMOMDPM given in [1] and TFN-DMOPDRM given in [32]; however, the results of selection and ranking of the set of the alternative decisionmaking objects based on the three model algorithms are consistent, which have the same optimal solution. The TFNDMORSPM algorithm presented in the paper not only combines the information of relative similarity degree of pairwise comparison between attribute values but also better show the rationality and validity of the model compared with other

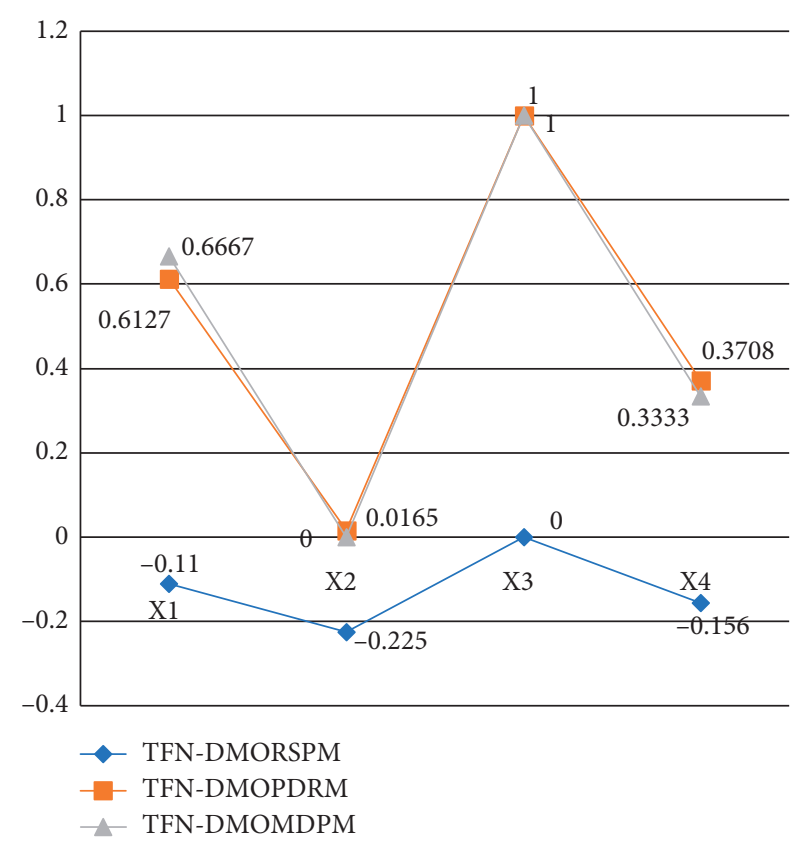

FIgURE 3: Geometrical comparison of TFN-DMORSPM, TFNDMOPDRM, and TFN-DMOMDPM.

existing methods. When the attribute utility value data is with high similarity degree, the TFN-DMORSPM algorithm can reduce the error of information aggregation and further increase the discrimination between alternative decision-making objects. It is beneficial to the accurate calculation, aggregation, and fusion of attribute utility data, as well as the picking and sorting of decision-making objects.

\section{Conclusion}

The attribute weighting rule based on TFN-DMORSPM is one of the key issues in UMADM research; it is a new weighting idea from the perspective of picking over and sorting the alternative decision-making objects: when the comprehensive relative similarity value of attribute measurement value data between decision-making objects is large (the floating of attribute measurement value data is small), the attribute plays a small role in picking over and sorting alternative decision-making objects, and the attribute weight should be reduced; otherwise, the attribute plays a greater role in picking over and sorting alternative decision-making objects, and the attribute weight should be increased.

In this paper, the UMADM problem with unknown attribute weights and no decision objects preference has been addressed by using the abovementioned optimization weighting idea and the advantage relation theories to comparative relative similarity degree of triangular fuzzy number. The main heighlights can be summarized into the following three aspects. (1) From the perspective of similarity degree measurement of triangular fuzzy number, some new definition formulas of max-relative similarity and min-relative similarity of normalized triangular fuzzy number and max-relative similarity degree and min-relative similarity degree of alternative decision-making 
objects have been given. By applyingthese formulas to the single target optimization problem of triangular fuzzy number-based decision-making objects relative similarity programming model, and the optimal measurement formula of attribute weight has been obtained. (2) Some results of the advantage relation theories to comparative relative similarity degree of triangular fuzzy number have been given. the equivalence relations between the dominant values of triangular fuzzy numbers and comparative maxrelative similarity degree values of triangular fuzzy number with the same ideal point or comparative min-relative similarity degree value of triangular fuzzy number with the same ideal point, and attribute values of small element, special element, and large element of triangular fuzzy number have beenrevealed. The equivalence relations of the dominant values of alternative decision-making objects and comparative max-relative similarity degree values of alternative decision-making objects with the same ideal point or comparative min-relative similarity degree value of alternative decision-making objects with the same ideal point and attribute values sequence and values of small element, special element, and large element of alternative decision-making objects have been presented. (3) The similarity degree model and algorithm in [33] have been improved. The comparative overall relative similarity degree between the alternative decision objects and the ideal optimal decision-making objects have been proposed to pick over and sort the set of the alternative decisionmaking objects; based on which the triangular fuzzy number-based decision-making object relative similarity programming model algorithm has been obtained.

On the basis of distinguishing the definition of triangular fuzzy number comparison max-relative similarity degree and min-relative similarity degree, this paper has investigatedthe related properties and application in the construction of attribute weight model. We have put forward the relation theories to comparative relative similarity degree of triangular fuzzy number constructing a triangular fuzzy number-based decisionmaking object relative similarity programming model and proposing a new model algorithm for the relative similarity programming of triangular fuzzy number-based multiple attribute decision-making alternatives by aggregating and merging the overall relative similarity degree information. The follow-up work can be focused on carrying out the research of the consistency correction algorithm around the relation of overall relative similarity degree and further developing new methods and solving the uncertain multiattribute decision-making problem.

\section{Data Availability}

The paper uses the example of scheduling problem about carrier-based aircraft models in literature [34] to analyze.

\section{Conflicts of Interest}

The authors declare that there are no conflicts of interest regarding the publication of this paper.

\section{Acknowledgments}

This work was supported by the National Social Science Foundation of China (Grant no. 20BGL132).

\section{References}

[1] Z. S. Xu, Uncertain Multiple Attribute Decision Making Methods and Applications, Tsinghua University Press, Beijing, China, 2004.

[2] P. Wang, P. Meng, and B. Song, "Response surface method using grey relational analysis for decision making in weapon system selection," Journal of Systems Engineering and Electronics, vol. 25, no. 2, pp. 265-272, 2014.

[3] L. Wu, S. Liu, and Y. Yang, "A model to determine OWA weights and its application in energy technology evaluation," International Journal of Intelligent Systems, vol. 30, no. 7, pp. 798-806, 2015.

[4] L. Tian, J. Luo, Y. F. Zeng et al., "Modeling and algorithms for multiagent communication through interactive dynamic influence diagrams," Applied Artificial Intelligence, vol. 30, no. 4, pp. 352-377, 2016.

[5] H. S. Kan, Y. B. Lu, Y. Le et al., "Agent-based computational model for project transaction governance," Systems Engineering-Theory \& Practice, vol. 37, no. 4, pp. 352-377, 2017.

[6] C. M. Cheng, X. Gao, D. Cheng et al., "Research on operational efficiency evaluation of anti-tank missile weapon system based on combination weighting," System Engineering Theory and Practice, vol. 38, no. 1, pp. 241-251, 2018.

[7] Z. L. Huang, Study on Interval Number-based and Triangular Fuzzy Number- based Uncertain Multi-Attribute DecisionMaking, Xiamen University, Xiamen, China, 2016.

[8] P. J. M. Van Laarhoven and W. Pedrycz, "A fuzzy extension of Saaty's priority theory," Fuzzy Sets and Systems, vol. 11, no. 13, pp. 229-241, 1983.

[9] E. Herrera-Viedma, F. Herrera, F. Chiclana, and M. Luque, "Some issues on consistency of fuzzy preference relations," European Journal of Operational Research, vol. 154, no. 1, pp. 98-109, 2004.

[10] Y.-M. Wang and C. Parkan, "Multiple attribute decision making based on fuzzy preference information on alternatives: ranking and weighting," Fuzzy Sets and Systems, vol. 153, no. 3, pp. 331-346, 2005.

[11] M. K. Sayadi, M. Heydari, and K. Shahanaghi, "Extension of VIKOR method for decision making problem with interval numbers," Applied Mathematical Modelling, vol. 33, no. 5, pp. 2257-2262, 2009.

[12] R. Kamran, S. R. Sara, N. Salman et al., "Evaluating performance of Iranian cement firms using an integrated fuzzy AHP-VIKOR method," Applied Mathematical Modelling, vol. 38, no. 21-22, pp. 5033-5046, 2014.

[13] Y. Sun, P. Y. Yao, L. J. Wan et al., "Multiple attribute decision making method based on weights aggregation and relative dominance relation," Control and Decision, vol. 32, no. 2, pp. 317-322, 2017.

[14] Z. L. Huang and J. Luo, "Relative similarity programming model for decision making objects with multiple criteria values as interval number," Systems Engineering-Theory \& Practice, vol. 39, no. 3, pp. 766-775, 2019.

[15] X. Chen, Z. L. Huang, and J. Luo, "Approach for triangular fuzzy number-based uncertain multi-attribute decision 
making based on relative similarity degree relation," Control and Decision, vol. 31, no. 12, pp. 2232-2240, 2016.

[16] H. A. Zhou and S. Y. Liu, "Method of uncertain multi-attribute decision-making based on quadratic programming and relative superiority degree," Systems Engineering and Electronics, vol. 29, no. 4, pp. 555-562, 2007.

[17] Y. Liu, J. Forrest, S. F. Liu et al., "Multi-objective grey target decision-making based on prospect theory," Control and Decision, vol. 28, no. 3, pp. 345-350, 2013.

[18] G.-W. Wei, "Grey relational analysis method for 2-tuple linguistic multiple attribute group decision making with incomplete weight information," Expert Systems with Applications, vol. 38, no. 5, pp. 4824-4828, 2011.

[19] Y. C. Gong, Z. Y. Ren, F. Ding et al., "Grey relation-projection pursuit dynamic cluster method for multiattribute decision making assessment with trapezoidal intuitionistic fuzzy numbers," Control and Decision, vol. 30, no. 7, pp. 1333-1339, 2015.

[20] X. H. Xu, P. Wang, and C. G. Cai, "Linguistic multi-attribute large group decision-making method based on similarity measurement of cloud model," Control and Decision, vol. 32, no. 3, pp. 459-466, 2017.

[21] L. Zhu, C. X. Zhu, and X. Z. Zhang, "Method for hesitant fuzzy multi-attribute decision making based on rough sets," Control and Decision, vol. 29, no. 7, pp. 1335-1339, 2014.

[22] H. Garg and D. Rani, "Some generalized complex intuitionistic fuzzy aggregation operators and their application to multicriteria decision-making process," Arabian Journal for Science and Engineering, vol. 44, no. 3, pp. 2679-2698, 2019.

[23] H. Garg and D. Rani, "Robust averaging-geometric aggregation operators for complex intuitionistic fuzzy sets and their applications to MCDM process," Arabian Journal for Science and Engineering, vol. 45, no. 3, pp. 2017-2033, 2020.

[24] X. Peng and Y. Yang, "Fundamental properties of intervalvalued pythagorean fuzzy aggregation operators," International Journal of Intelligent Systems, vol. 31, no. 5, pp. 444-487, 2016.

[25] X. Gou, Z. Xu, and P. Ren, "The properties of continuous pythagorean fuzzy information," International Journal of Intelligent Systems, vol. 31, no. 5, pp. 401-424, 2016.

[26] N. Li, H. Garg, and L. Wang, "Some novel interactive hybridweighted aggregation operators with pythagorean fuzzy numbers and their applications to decision making (Article)," Mathematics, vol. 7, no. 12, 2019.

[27] K. Ullah, T. Mahmood, Z. Ali, and N. Jan, "On some distance measures of complex Pythagorean fuzzy sets and their applications in pattern recognition," Complex \& Intelligent Systems, vol. 6, no. 1, pp. 15-27, 2020.

[28] Y. Xing, R. Zhang, Z. Zhou, and J. Wang, "Some q-rung orthopair fuzzy point weighted aggregation operators for multi-attribute decision making," Soft Computing, vol. 23, no. 22, pp. 11627-11649, 2019.

[29] H. a. Garg, J. b. Gwak, T. c. Mahmood et al., "Power aggregation operators and VIKOR methods for complex q-rung orthopair fuzzy sets and their applications," Mathematics, vol. 8, no. 4, 2020.

[30] T. Mahmood and Z. Ali, "Aggregation operators and VIKOR method based on complex q-rung orthopair uncertain linguistic informations and their applications in multi-attribute decision making," Computational and Applied Mathematics, vol. 39, no. 4, 2020.

[31] H. Garg, Z. Ali, and T. Mahmood, "Algorithms for complex interval-valued q-rung orthopair fuzzy sets in decision making based on aggregation operators," AHP, and TOPSIS. Expert Systems, vol. 38, no. 1, 2021.

[32] Z. L. Huang, "Possibility degree relation model for decision making objects with multiple criteria values as triangular fuzzy number," Control and Decision, vol. 33, no. 11, pp. 1931-1940, 2018.

[33] J. Liu, S. F. Liu, X. Z. Zhou et al., "Research on multipleattribute decision making problems based on the similarity relationship," System Engineering and Electronics, vol. 33, no. 5, pp. 1069-1072, 2011.

[34] L. Y. Hu, H. J. Yuan, and Q. P. Wu, "Multiple attribute decision-making of triangular fuzzy number based on set pair analysis," Journal of Wuhan University of Technology (Information \& Management Engineering), vol. 37, no. 1, pp. 108-111, 2015. 